PREPARED For SUBMisSion to JCAP

\title{
Consequences of neutrino self-interactions for weak decoupling and big bang nucleosynthesis
}

\section{E. Grohs, ${ }^{a, 1}$ George M. Fuller, ${ }^{b}$ Manibrata Sen, ${ }^{a, c}$}

\author{
${ }^{a}$ Department of Physics, University of California, Berkeley, Berkeley, California 94729, USA \\ ${ }^{b}$ Department of Physics, University of California, San Diego, La Jolla, California 92093-0319, \\ USA \\ ${ }^{c}$ Department of Physics and Astronomy, Northwestern University, Evanston, Illinois 60208- \\ 3112 , USA
}

\section{E-mail: egrohs@berkeley.edu}

\begin{abstract}
We calculate and discuss the implications of neutrino self-interactions for the physics of weak decoupling and big bang nucleosynthesis (BBN) in the early universe. In such neutrino-sector extensions of the standard model, neutrinos may not free-stream, yet can stay thermally coupled to one another. Nevertheless, the neutrinos exchange energy and entropy with the photon, electron-positron, and baryon component of the early universe only through the ordinary weak interaction. We examine the effects of neutrino self-interaction for the primordial helium and deuterium abundances and $N_{\text {eff }}$, a measure of relativistic energy density at photon decoupling. These quantities are determined in, or may be influenced by, the physics in the weak decoupling epoch. Self-interacting neutrinos have been invoked to address a number of anomalies, including as a possible means of ameliorating tension in the Hubble parameter. Our calculations show that surprisingly subtle changes in BBN accompany some of these neutrino self-interaction schemes. Such minute signals require highprecision measurements, making deuterium the best abundance for BBN constraints in the models explored here.
\end{abstract}

Keywords: neutrino theory, cosmological neutrinos, big bang nucleosynthesis, Hubble parameter tension

\footnotetext{
${ }^{1}$ Corresponding author.
} 


\section{Contents}

1 Introduction $\quad 1$

2 Overview of Big Bang Nucleosynthesis $\quad 4$

2.1 Physics 4

2.2 Numerics 5

$\begin{array}{lll}2.2 .1 & \text { Computational Code } & 7\end{array}$

$\begin{array}{lll}\text { 2.2.2 Results in the Standard Scenario } & 7\end{array}$

3 Lepton Symmetric Initial Conditions $\quad 7$

3.1 Neutrino transport with Self-Interactions 7

3.2 Results 9

3.2.1 Neutrino Spectra 9

$\begin{array}{ll}3.2 .2 & \text { Primordial Abundances } \\ \end{array}$

4 Dark Radiation Addition $\quad 11$

4.1 Dark Radiation Parameter 11

4.2 Results 12

$\begin{array}{lll}\text { 4.2.1 Neutrino Spectra } & 12\end{array}$

$\begin{array}{lll}4.2 .2 & \text { Primordial Abundances } & 14\end{array}$

5 Lepton Asymmetric Initial Conditions $\quad 14$

5.1 Differences in Implementation with Symmetric Scenario 14

$\begin{array}{lll}5.2 & \text { Results } & 15\end{array}$

$\begin{array}{lll}\text { 5.2.1 Neutrino Spectra } & 15\end{array}$

5.2.2 Primordial Abundances 16

6 Conclusions $\quad 17$

$\begin{array}{lll}6.1 & \text { Summary } & 17\end{array}$

$\begin{array}{lll}6.2 & \text { Discussion } & 18\end{array}$

\section{Introduction}

In this paper we report detailed calculations of the evolution of the early universe when selfinteracting neutrino standard model extensions are adopted. Though many properties of the neutrinos have been measured, including the mass-squared differences and three of the four parameters in the unitary transformation between the neutrino weak interaction (flavor) basis and the mass basis [1], much remains unexplored and mysterious in this sector of particle physics. Indeed, many extensions of the standard model in the neutrino sector have been proposed, and for a variety of purposes. Standard model extensions that posit interactions among the neutrinos only - sometimes termed self-interactions or "secret" interactions - are notoriously difficult to constrain in experiments. However, two astrophysical venues perhaps offer a window into this physics. Both the early universe [2] and collapsing or merging compact objects [3] at some point have their energy and dynamics influenced by neutrinoneutrino scattering. The former venue is a promising test bed for this physics because of 
the advent of high precision cosmology; the latter because of the burgeoning capabilities in multi-messenger astronomy. Cosmological invocations of Self-Interacting Neutrino (SI $\nu$ ) models include attempts to: reconcile sterile neutrino interpretations of neutrino anomalies with cosmological bounds [4-7]; produce sterile-neutrino dark matter [8, 9]; suppress active neutrino free-streaming [2]; and act as a principal participant in schemes crafted to reconcile discordant measurements of the Hubble parameter $[10,11]$. SI $\nu$ can also put constraints on models of inflation $[12,13]$ and influence flavor evolution of dense neutrino streams in compact objects $[14-16]$.

There are many standard model extensions in the neutrino sector which incorporate neutrino self-interactions. Some of these, for example, singlet Majoron and Familon models [17-19] envision a spontaneously broken symmetry with a Goldstone boson which mediates neutrino self-interactions. In these models, exchange of this boson engenders flavor-changing interactions among the neutrinos with interaction strengths that can be many orders of magnitude stronger than the standard model weak interaction. Here we consider a scalarexchange model based off of Eq. (2) in Ref. [9]. The interaction Lagrangian $\mathcal{L}_{\text {int }}$ has effective couplings $G_{i j}$ between neutrino species $i$ and $j$

$$
\mathcal{L}_{\text {int }}=g_{i j} \overline{\nu_{i L}^{c}} \nu_{j L} \varphi+g_{i j} \overline{\nu_{i L}} \nu_{j L}^{c} \varphi, \quad G_{i j}=\frac{g_{i j}^{2}}{m_{\varphi}^{2}},
$$

where $\nu_{L}$ is a left-handed-neutrino Dirac spinor and $m_{\varphi}$ is the rest mass of the scalar exchange particle $\varphi$. The superscript $c$ on the Dirac spinor indicates charge conjugation. With the model in Eq. (1.1), neutrinos cannot undergo spin-flips at order $g^{2}$. Spin-flips are possible at order $g^{3}$, but such a mechanism requires other features of an ultraviolet-complete theory, e.g., Eq. (1.3) in Ref. [20]. The overall coupling $G_{i j}$ is analogous to the Fermi constant $G_{\mathrm{F}}$ in the standard weak interaction, so that typical cross sections in our adopted schematic self-interaction model will scale with energy as $\sigma_{i j} \sim G_{i j}^{2} E_{\nu}^{2}$. References [20,21] study how to embed the operator in Eq. (1.1) into UV-complete models.

In this work we will consider only processes which couple neutrinos to other neutrinos, albeit with flavor changing currents. Specifically, we include $\nu \nu$ and $\overline{\nu \nu} s$-channel processes and $\nu \bar{\nu} t$-channel processes, where the later manifests in lepton-asymmetric systems. We will not consider processes which convert a neutrino into an anti-neutrino. It is most convenient to represent the couplings in our generic model in flavor-basis matrix form:

$$
\left[g_{i j}\right]=\left(\begin{array}{lll}
g_{e e} & g_{e \mu} & g_{e \tau} \\
g_{\mu e} & g_{\mu \mu} & g_{\mu \tau} \\
g_{\tau e} & g_{\tau \mu} & g_{\tau \tau}
\end{array}\right) \Rightarrow g\left(\begin{array}{lll}
1 & 1 & 1 \\
1 & 1 & 1 \\
1 & 1 & 1
\end{array}\right)
$$

Here the flavor off-diagonal entries produce flavor-changing couplings. The second matrix represents the coupling scheme we adopt for our early universe weak decoupling and Big Bang Nucleosynthesis (BBN) calculations. We employ an overall coupling strength $g$ and equal strength in all neutrino-neutrino scattering channels, including those which change flavor. Our generic model then has two independently specifiable quantities: $g$ and $m_{\varphi}$.

We will further restrict the range of neutrino self-interaction parameters we actually simulate by restricting the mass of the scalar $m_{\varphi}$ to be much larger than the range of temperatures $T$ that our weak decoupling neutrino transport and nuclear reaction calculations cover. In other words, we will assume $m_{\varphi} \gg T$. The temperature range of our calculations span the entire weak decoupling and BBN epoch. Generously, and therefore conservatively, we 
take this range to be $30 \mathrm{MeV}>T>1 \mathrm{keV}$. Restricting the scalar mass to be $m_{\varphi} \gg 30 \mathrm{MeV}$ means that we will not have to be concerned with on-shell production and population of these particles during weak decoupling. Moreover, though the scalar particles may be produced in significant abundance early on, for example at temperatures $T>m_{\varphi}$, we will consider only coupling strengths $g$ sufficiently large to keep these particles in equilibrium with the neutrino component. This guarantees that the scalar particles will have disappeared by the beginning time of our simulations. Such relatively large couplings also preclude out-of-equilibrium scalar particle decays and concomitant entropy generation during weak decoupling and BBN. We note, however, that cosmology does not provide the only constraint on SI $\nu$ models [22].

Finally, we assume that the overall coupling $g$ is large enough to produce efficient energy and flavor exchange among the neutrinos. We compare the self-interacting neutrino scattering rate, $\Gamma_{\mathrm{SI}}$, to the Hubble expansion rate, $H$, to estimate when the neutrinos decouple from themselves

$$
\Gamma_{\mathrm{SI}} \sim H \Longrightarrow\left(\frac{g^{2}}{m_{\varphi}^{2}}\right)^{2}\left\langle E^{2}\right\rangle n_{\nu} \sim \sqrt{\frac{8 \pi}{3} \frac{\pi^{2}}{30} g_{\star}} \frac{T^{2}}{m_{\mathrm{pl}}},
$$

where $\left\langle E^{2}\right\rangle$ is the average square energy, $n_{\nu}$ is the number density of neutrinos, $g_{\star}$ is the relativistic degrees of freedom [23], and $m_{\mathrm{pl}}$ is the Planck mass. If we approximate $\left\langle E^{2}\right\rangle \approx T^{2}$ and use Fermi-Dirac (FD) statistics for 3 flavors of neutrinos to deduce $n_{\nu}=3 \times 3 \zeta(3) T^{3} /\left(4 \pi^{2}\right)$, we can solve for $g$

$$
g \sim 5.2 \times 10^{-2}\left(\frac{m_{\varphi}}{30 \mathrm{MeV}}\right)\left(\frac{1 \mathrm{keV}}{T}\right)^{3 / 4}\left(\frac{g_{\star}}{3.36}\right)^{1 / 8}
$$

For a decoupling temperature $T \simeq 1 \mathrm{keV}$, we estimate an effective coupling $G_{\text {eff }} \equiv g^{2} / m_{\varphi}^{2} \simeq$ $3.0 \times 10^{-6} \mathrm{MeV}^{-2}$, in line with the models proposed in Ref. [11] to alleviate the Hubble parameter tension [24]. For $m_{\varphi}=30 \mathrm{MeV}$, kaon decay [25, 26] rules out a universal coupling in this range [22], implying the mediator mass must be larger than $\sim 100 \mathrm{MeV}$. With our assumption that $m_{\varphi} \gg T$, this "self-equilibrium" in the neutrino component is effected via two-to-two neutrino scattering processes on timescales short compared to standard chargedand neutral-current weak interaction times, and for the entire temperature range employed in our calculations.

The neutrino component will be essentially instantaneously self-coupled and energyand flavor-equilibrated in this model. However, the neutrinos will exchange energy and entropy with the photon-electron-positron-baryon plasma only through the ordinary standard model weak interactions. This sets up a formidable transport problem wherein we must follow self-consistently the flow of energy and entropy between the various components while simultaneously calculating the strong, electromagnetic and weak interactions that determine the quantities that can be probed, i.e., the light element abundances and relic neutrino energy density. The latter quantity derives from the inferred radiation energy density $\rho_{\text {rad }}$ at the photon decoupling epoch, $T \approx 0.2 \mathrm{eV}$, and is parameterized by $N_{\text {eff }}$, defined through

$$
\rho_{\text {rad }}=\left[2+\frac{7}{4}\left(\frac{4}{11}\right)^{4 / 3} N_{\text {eff }}\right] \frac{\pi^{2}}{30} T^{4} .
$$

The outline of this paper is as follows. To describe the SI $\nu$ scenario, it will prove helpful to give a brief review of BBN in Sec. 2. Subsections 2.1 and 2.2 describes the physics and numerics of BBN with particular emphasis on the evolution of the neutrino seas. In Sec. 
3, we present our first $\mathrm{SI} \nu$ results with neutrino-anti-neutrino symmetric initial conditions. Subsection 3.1 specifies the physics and computational model of $\mathrm{SI} \nu$, and Subsection 3.2 gives results. To further investigate self-interactions during BBN, we provide two extensions to standard BBN with an addition of dark radiation and neutrino-anti-neutrino asymmetric initial conditions in Secs. 4 and 5. We summarize our results and discuss further implications of self-interacting neutrinos in Sec. 6. Throughout this paper we set $c=\hbar=k_{B}=1$.

\section{Overview of Big Bang Nucleosynthesis}

We direct the reader to Refs. [23, 27-29] for a treatment on the basic physics of BBN, and Ref. [30] for an overview of the current status of the field. We give a brief review on the relevant topics of standard BBN here to contrast the standard scenario with that of $\mathrm{SI} \nu$.

\subsection{Physics}

Big Bang Nucleosynthesis is a succession of out-of-equilibrium freeze-outs. The first freezeout epoch is weak decoupling, when the neutrinos decouple from the charged leptons and themselves. Weak decoupling commences at temperature scales of a few MeV. The weak interaction mediates an exchange of energy from the electrons and positrons into the neutrino seas

$$
\begin{aligned}
& \nu+e^{ \pm} \leftrightarrow \nu+e^{ \pm}, \\
& \nu+\bar{\nu} \leftrightarrow e^{-}+e^{+} .
\end{aligned}
$$

Both of the above processes include charged-current and neutral-current interactions. Electronflavor neutrinos will experience both charged and neutral-current interactions, while $\mu$ and $\tau$-flavor only experience neutral-current. As a result, more heat flows into the electron-flavor sector than either the $\mu$ or $\tau$. Three other neutral-current interactions redistribute energy among the neutrino seas

$$
\begin{aligned}
\nu_{i}+\nu_{j} & \leftrightarrow \nu_{i}+\nu_{j}, \\
\nu_{i}+\bar{\nu}_{j} & \leftrightarrow \nu_{i}+\bar{\nu}_{j}, \\
\nu_{i}+\bar{\nu}_{i} & \leftrightarrow \nu_{j}+\bar{\nu}_{j} .
\end{aligned}
$$

The above three interactions lead to a "flavor-equilibration" (see Figs. [7] and [8] in Ref. [31]) among the electron and $\mu / \tau$ flavors.

Weak decoupling concludes at a temperature of a few 10's of keV. The neutrinos will also decouple from the baryons during the Weak Freeze-Out (WFO) epoch. WFO and weak decoupling have a significant overlap in time. However, due to the large number of neutrinos compared to baryons, WFO and weak decoupling are not simultaneous. We monitor the change in the neutron-to-proton ratio of the baryons by calculating six neutron-to-proton rates, schematically given as

$$
\begin{aligned}
\nu_{e}+n & \leftrightarrow p+e^{-}, \\
e^{+}+n & \leftrightarrow p+\bar{\nu}_{e}, \\
n & \leftrightarrow p+\bar{\nu}_{e}+e^{-} .
\end{aligned}
$$

We do not consider the energy and entropy flow from the baryon component into the neutrino seas as this is a small change compared to the entropies of the neutrino seas and plasma. 
As the temperature continues to decrease, the electron-positron pairs will annihilate into photons during the eponymous electron-positron annihilation epoch. The last freezeout epoch is Nuclear Freeze-Out (NFO), when the nuclear abundances go out of nuclear statistical equilibrium and obtain their primordial values. For our purposes, the physics in $e^{ \pm}$-annihilation and NFO are identical in the standard and $\mathrm{SI} \nu$ scenarios. Although the physics is distinct, the preceding four freeze-out epochs are not temporally distinct. They overlap with one another and require computational tools to model the physics.

\subsection{Numerics}

At high temperatures, weak interactions keep the neutrinos in thermal and chemical equilibrium with the electromagnetic plasma, i.e., $T_{\nu}=T$. The time derivative of the plasma temperature $T$ is based off of Eq. (D28) in [32]

$$
\frac{d T}{d t}=-3 H\left(\frac{d \rho_{\mathrm{pl}}}{d T}\right)^{-1}\left(\rho_{\mathrm{pl}}+P_{\mathrm{pl}}-\left.\frac{1}{3 H} \frac{d Q}{d t}\right|_{T}\right)
$$

where $\rho_{\mathrm{pl}}$ is the energy density of the plasma (less baryons), $P_{\mathrm{pl}}$ is the pressure exerted by all plasma components, $d Q /\left.d t\right|_{T}$ is the plasma heat gained/lost from microphysics, and $d \rho_{\mathrm{pl}} / d T$ is the temperature derivative of the plasma components (including baryons) [33]. When calculating the electromagnetic energy densities, we implement finite temperature QED effects to the electromagnetic equation of state as explained in [34-36] (see Ref. [37] for a detailed analysis of the finite-temperature QED problem in the early universe). At high temperatures, the plasma consists of photons, electrons, positrons, neutrinos, and baryons.

We begin the weak decoupling process at $10 \mathrm{MeV}$ by dissociating the electromagnetic components from the neutrino seas. The electromagnetic plasma temperature continues to evolve according to Eq. (2.9) without neutrinos. In the standard scenario, we evolve the neutrino occupation fractions $f_{i}(\epsilon), i=e, \mu, \tau$ using a weak-interaction collision operator

$$
\begin{aligned}
\left.\mathcal{C}_{j}\left[f_{i}\left(\epsilon_{1}\right)\right]\right|_{\epsilon_{1}=E_{1} / T_{\mathrm{cm}}}=\frac{1}{2 E_{1}} \int & \frac{d^{3} p_{2}}{(2 \pi)^{3} 2 E_{2}} \frac{d^{3} p_{3}}{(2 \pi)^{3} 2 E_{3}} \frac{d^{3} p_{4}}{(2 \pi)^{3} 2 E_{4}}\left\langle\left|\mathcal{M}_{j}\right|^{2}\right\rangle \delta^{(4)}\left(P_{1}+P_{2}-P_{3}-P_{4}\right) \\
& \times\left[\left(1-f^{(1)}\right)\left(1-f^{(2)}\right) f^{(3)} f^{(4)}-f^{(1)} f^{(2)}\left(1-f^{(3)}\right)\left(1-f^{(4)}\right)\right],
\end{aligned}
$$

where $j$ indicates the collision type corresponding to a reaction in Eqs. $(2.1)-(2.5)$. $P_{k}$ is the four momentum for particle $k$ and $\left\langle\left|\mathcal{M}_{j}\right|^{2}\right\rangle$ is the amplitude for reaction $j$ (see Table I in Ref. [31]). We use the shorthand notation $f^{(k)} \equiv f\left(\epsilon_{k}\right), k=1,2,3,4$, to denote the fermion occupation numbers for either neutrinos or charged leptons, where the latter is always a FD distribution

$$
f_{e^{ \pm}}(\epsilon)=\frac{1}{\exp \left(\epsilon \frac{T_{\mathrm{cm}}}{T} \mp \phi_{e}\right)+1}
$$

and $\phi_{e}$ is the electron degeneracy parameter defined below. There exists an implicit subscript on $f^{(k)}$ in Eq. (2.10) to denote either the flavor (neutrino or antineutrino) or the charged lepton (electron or positron) depending on the collision $j$. We can calculate the heat flow from the plasma to the neutrino seas by integrating over the collision terms involving the charged leptons

$$
\left.\frac{\partial \rho_{\nu}}{\partial t}\right|_{a}=\frac{T_{\mathrm{cm}}^{4}}{2 \pi^{2}} \sum_{i=1}^{6} \int d \epsilon \epsilon^{3}\left\{\mathcal{C}_{\nu e}\left[f_{i}(\epsilon)\right]+\mathcal{C}_{e^{-} e^{+}}\left[f_{i}(\epsilon)\right]\right\}
$$


Equation (2.12) communicates the change to the plasma using the $d Q /\left.d t\right|_{T}$ term in Eq. (2.9)

1. Therefore, the final expression for the heat gain/lost due to microphysics is

$$
\left.\frac{d Q}{d t}\right|_{T}=\left.\frac{\partial \rho_{\mathrm{pl}}}{\partial t}\right|_{\mathrm{Nuc}}-\left.\frac{\partial \rho_{\nu}}{\partial t}\right|_{a}
$$

where the first term is the heat gained from nucleosynthesis, and the minus sign indicates the plasma loses energy to the neutrino seas during weak decoupling.

The only terms in the evolution for the neutrino occupation numbers are the collision operators, i.e.,

$$
\frac{d f_{i}(\epsilon)}{d t}=\sum_{j} \mathcal{C}_{j}\left[f_{i}(\epsilon)\right]
$$

We only evolve the occupation numbers of the neutrinos after the universe has reached a temperature of $10 \mathrm{MeV}$. When the neutrinos decouple in the standard scenario, they do not follow an equilibrium distribution and so there is no need to evolve temperatures or degeneracy parameters of the neutrino components. $T_{\mathrm{cm}}$ acts as an energy scale for the neutrinos so that we may calculate energy density quantities, e.g., Eq. (2.12).

Equation (2.9) is derived from conservation of energy [32], but does not conserve particle number. However, we do implement conservation of charge by evolving the electron degeneracy parameter

$$
\phi_{e} \equiv \frac{\mu_{e^{-}}}{T},
$$

for electron chemical potential $\mu_{e^{-}}$. The positron chemical potential is equal in magnitude and opposite in sign if we assume chemical equilibrium between the electrons and positrons. We evolve $\phi_{e}$ with time by ensuring electrons and positrons maintain FD distributions and the difference of the two number densities is equal to the number density of protons (both free and those bound in nuclei)

$$
n_{e^{-}}-n_{e^{+}}=n_{b} \sum_{i} Z_{i} Y_{i}
$$

for baryon number density $n_{b}$, atomic numbers $Z_{i}$, and nuclear abundances $Y_{i} \equiv n_{i} / n_{b}$. We do not explicitly evolve the degeneracy parameters for the nuclei, but instead evolve their abundances $Y_{i}$ through the nuclear reaction network with an example equation

$$
\frac{d Y_{i}}{d t}=\sum_{j, k, l}\left\{\frac{Y_{k}^{N_{k}} Y_{l}^{N_{l}}}{N_{k} ! N_{l} !}[k l]_{i j}-\frac{Y_{i}^{N_{i}} Y_{j}^{N_{j}}}{N_{i} ! N_{j} !}[i j]_{k l}\right\},
$$

where $N_{i, j, k, l}$ are the number of each nucleus in the reaction $i(j, k) l,[k l]_{i j}$ is the reaction rate coefficient to create nucleus $i$, and $[i j]_{k l}$ is the reverse reaction rate coefficient [32]. The comoving number of protons changes as the weak-charged-current reactions change neutrons into protons, and vice-versa. We use Eqs. [19-24] in Ref. [38] to calculate the rates, which are input into Eq. (2.17). Our rate expressions are single integrals over the neutrino energy. We can use either FD expressions or out-of-equilibrium occupation fractions to characterize the neutrino distribution.

\footnotetext{
${ }^{1}$ We have switched which variable we hold constant in our derivatives with respect to time, either the plasma temperature $T$ or the scale factor $a$. Although the distinction is important in a thermodynamic sense, $T$ can be parameterized using $a$ implying $d \rho_{\nu} /\left.d t\right|_{a}=d \rho_{\nu} /\left.d t\right|_{T}$.
} 
The last quantity to evolve is the scale factor with the Friedmann Equation

$$
H \equiv \frac{1}{a} \frac{d a}{d t}=\sqrt{\frac{8 \pi}{3 m_{\mathrm{pl}}^{2}} \rho_{\mathrm{tot}}},
$$

which defines the Hubble expansion rate $H . m_{\mathrm{pl}}$ is the Planck mass, and $\rho_{\text {tot }}$ is the total energy density of the universe: photons, electrons, positrons, neutrinos, baryons/nuclei, dark radiation, dark matter, and dark energy. In practice, we ignore the contribution from dark matter and dark energy as they are negligible during the BBN epoch.

\subsubsection{Computational Code}

Public BBN codes exist, see Refs. [39-41]. We use our code BURST based off of Refs. [31, 42]. We integrate the coupled ordinary differential equations for $T, \phi_{e}, a,\left\{Y_{i}\right\},\left\{f_{i}\left(\epsilon_{j}\right)\right\}$ with respect to the coordinate time $t$. Our calculations commence at $T_{\mathrm{cm}}=30 \mathrm{MeV}$ and terminate at $T_{\mathrm{cm}}=1 \mathrm{keV}$. We begin calculating the neutrino collision integrals at $T_{\mathrm{cm}}=10 \mathrm{MeV}$. We integrate the nuclear reaction network at all times.

\subsubsection{Results in the Standard Scenario}

In the standard scenario, we find $N_{\text {eff }}=3.044$, and the following abundances

$$
\begin{aligned}
& Y_{\mathrm{P}}=0.2478, \\
& \mathrm{D} / \mathrm{H}=2.624 \times 10^{-5},
\end{aligned}
$$

for a baryon density $\omega_{b}=0.02226$, consistent with Ref. [43], and a neutron lifetime $\tau_{n}=$ $885.1 \mathrm{~s}$. Our value for primordial lithium is ${ }^{7} \mathrm{Li} / \mathrm{H}=4.375 \times 10^{-10}$. We caution that the abundances are dependent on the nuclear reaction rates, and so the above quantities should be taken as a baseline when compared to the SI $\nu$ scenario, and not absolute predictions.

\section{Lepton Symmetric Initial Conditions}

\subsection{Neutrino transport with Self-Interactions}

With self-interactions, we need to differentiate between two different epochs/phenomena. We will term the process of neutrinos decoupling from the electromagnetic plasma "weak decoupling," in accordance with the terminology used in the standard scenario. When neutrinos decouple from each other, we will call this process "neutrino decoupling." Weak and neutrino decoupling are contemporaneous in the standard model, but occur on vastly different time/energy scales in the SI $\nu$ scenario. In our model, we assume that the self-interactions keep the neutrinos in a FD distribution throughout weak decoupling. This neutrino distribution differs from the electron/positron FD distribution in both number and energy density. For lepton-symmetric initial conditions, the FD distribution is identical for neutrinos and anti-neutrinos and we characterize it using a temperature $T_{\nu}$ and degeneracy quantity

$$
\eta_{\nu}=\frac{\mu_{\nu}}{T_{\nu}}
$$

for neutrino chemical potential $\mu_{\nu}$. As the universe evolves through weak decoupling, there will be a transfer of both energy and particle number to the neutrino seas through the 
weak interactions. The self interactions only thermalize the neutrino seas by exchanging energy among the constituent particles. These interactions neither create nor destroy neutrino number through communication with the electromagnetic plasma. As a result, energy and number are conserved in the $\mathrm{SI} \nu$ scenario, unlike how we conserve energy and charge for the electromagnetic plasma components in Eqs. (2.9) and (2.16). We must evolve $T_{\nu}$ and $\eta_{\nu}$ using the definitions of energy and number density

$$
\left.\begin{array}{l}
n\left(T_{\nu}, \eta_{\nu}\right)=N_{\nu} \frac{T_{\nu}^{3}}{2 \pi^{2}} \int d \epsilon \epsilon^{2} f^{(\mathrm{eq})}\left(\epsilon ; \eta_{\nu}\right) \\
\rho\left(T_{\nu}, \eta_{\nu}\right)=N_{\nu} \frac{T_{\nu}^{4}}{2 \pi^{2}} \int d \epsilon \epsilon^{3} f^{(\mathrm{eq})}\left(\epsilon ; \eta_{\nu}\right)
\end{array}\right\} \Longrightarrow\left\{\begin{array}{l}
T_{\nu}=T_{\nu}(n, \rho) \\
\eta_{\nu}=\eta_{\nu}(n, \rho)
\end{array}\right.
$$

where we write $T_{\nu}$ and $\eta_{\nu}$ as functions of $n$ and $\rho$, and the FD expression $f^{(\mathrm{eq})}\left(\epsilon ; \eta_{\nu}\right)$ is

$$
f^{(\mathrm{eq})}\left(\epsilon ; \eta_{\nu}\right)=\frac{1}{e^{\epsilon-\eta_{\nu}}+1}
$$

In Eq. (3.2), $N_{\nu}$ is the number of particle species and is equal to $6\left(\nu_{e}, \bar{\nu}_{e}, \nu_{\mu}, \bar{\nu}_{\mu}, \nu_{\tau}, \bar{\nu}_{\tau}\right)$ in the non-degenerate $\mathrm{SI} \nu$ scenario. If we add energy or number to the neutrino seas, we change both $T_{\nu}$ and $\eta_{\nu}$ via the definitions of energy and number density

$$
\frac{d \chi}{d t}=\frac{\partial \chi}{\partial T_{\nu}} \frac{d T_{\nu}}{d t}+\frac{\partial \chi}{\partial \eta_{\nu}} \frac{d \eta_{\nu}}{d t}
$$

where $\chi$ denotes either $\rho$ or $n$. Therefore, we find the time derivative of $T_{\nu}$ to be

$$
\frac{d T_{\nu}}{d t}=-H T_{\nu}+T_{\nu} \frac{n,\left.\eta \frac{\partial \rho}{\partial t}\right|_{a}-\left.3 T_{\nu} n \frac{\partial n}{\partial t}\right|_{a}}{4 \rho n,_{\eta}-9 T_{\nu} n^{2}},
$$

and the time derivative of $\eta_{\nu}$

$$
\frac{d \eta_{\nu}}{d t}=\frac{\left.4 \rho \frac{\partial n}{\partial t}\right|_{a}-\left.3 n \frac{\partial \rho}{\partial t}\right|_{a}}{4 \rho n,,_{\eta}-9 T_{\nu} n^{2}}
$$

where $\partial n /\left.\partial t\right|_{a}$ is the number density added from out-of-equilibrium weak decoupling, $\partial \rho /\left.\partial t\right|_{a}$ is the energy density added, and $n, \eta$ is the following expression

$$
n_{, \eta}=N_{\nu} \frac{T_{\nu}^{3}}{\pi^{2}} \int d \epsilon \epsilon f^{(\mathrm{eq})}\left(\epsilon ; \eta_{\nu}\right)
$$

We have written $T_{\nu}$ and $\eta_{\nu}$ as functions of energy and number density in Eq. (3.2). Although $\rho$ and $n$ have familiar meanings in an out-of-equilibrium system, temperature and chemical potential (i.e., degeneracy parameter) do not have the same thermodynamic meaning as they would in an equilibrium system. Simply stated, $T_{\nu}$ and $\eta_{\nu}$ are parameters for energy and number distributions. Equations (3.5) and (3.6) give the equations of motion for the two parameters and are consistent with the definitions of $n$ and $\rho$ in Eq. (3.2). The electromagnetic plasma acts as a heat source for the neutrinos, but the lack of a tight-coupling between the two systems precludes the equivalence of $T_{\nu}$ and $\eta_{\nu}$ with the thermodynamic 
variables of temperature and degeneracy parameter of the grand canonical ensemble. Nevertheless, we will colloquially refer to $T_{\nu}$ and $\eta_{\nu}$ as temperature and degeneracy parameter, respectively, throughout this manuscript.

There are two weak interaction collision terms we use in the $\mathrm{SI} \nu$ scenario

$$
\begin{aligned}
& \nu+e^{ \pm} \leftrightarrow \nu+e^{ \pm}, \\
& \nu+\bar{\nu} \leftrightarrow e^{-}+e^{+},
\end{aligned}
$$

with collision terms $\mathcal{C}_{\nu e}$ and $\mathcal{C}_{e^{-} e^{+}}$, respectively. $\mathcal{C}_{\nu e}$ and $\mathcal{C}_{e^{-} e^{+}}$are the same collision operators for the standard scenario of out-of-equilibrium neutrino spectra (see Appendix C in Ref. [31]). For our purposes, we do not evolve the individual occupation fractions, $f_{\nu_{i}}(\epsilon)$, of the neutrino spectra. Instead, we use the FD expression $f^{(\mathrm{eq})}\left(\epsilon ; \eta_{\nu}\right)$ for both terms and integrate over $\epsilon$ to find the total added neutrino number and energy density for a given time step. Note that $\mathcal{C}_{\nu e}$ preserves the neutrino number and changes the energy, while $\mathcal{C}_{e^{-}} e^{+}$changes both number and energy. Therefore, the number and energy density added during the out-of-equilibrium weak decoupling is

$$
\begin{aligned}
& \left.\frac{\partial n}{\partial t}\right|_{a}=\frac{T_{\nu}^{3}}{2 \pi^{2}} \sum_{i=1}^{6} \int d \epsilon \epsilon^{2} \mathcal{C}_{e^{-} e^{+}}\left[f^{(\mathrm{eq})}\left(\epsilon ; \eta_{\nu}\right)\right], \\
& \left.\frac{\partial \rho}{\partial t}\right|_{a}=\frac{T_{\nu}^{4}}{2 \pi^{2}} \sum_{i=1}^{6} \int d \epsilon \epsilon^{3}\left\{\mathcal{C}_{\nu e}\left[f^{(\mathrm{eq})}\left(\epsilon ; \eta_{\nu}\right)\right]+\mathcal{C}_{e^{-} e^{+}}\left[f^{(\mathrm{eq})}\left(\epsilon ; \eta_{\nu}\right)\right]\right\} .
\end{aligned}
$$

Equation (3.11) is a copy of Eq. (2.12) except we use $T_{\nu}$ instead of $T_{\mathrm{cm}}$ for the energy scale.

There is neither number nor energy density added to the neutrino seas after weak decoupling concludes. At this point, Eqs. (3.5) and (3.6) reduce to

$$
\begin{aligned}
& \frac{d T_{\nu}}{d t}=-H T_{\nu}, \\
& \frac{d \eta_{\nu}}{d t}=0,
\end{aligned}
$$

which are the free-streaming conditions for relativistic particles.

\subsection{Results}

\subsubsection{Neutrino Spectra}

Figure 1 gives the relative change of the neutrino occupation fractions from non-degenerate FD equilibrium, i.e.,

$$
\delta f(\epsilon) \equiv \frac{f(\epsilon)-f^{(\mathrm{eq})}(\epsilon ; 0)}{f^{(\mathrm{eq})}(\epsilon ; 0)},
$$

versus the dimensionless comoving energy. The dashed lines are the out-of-equilibrium neutrino spectra for $e$-flavor neutrinos (green) and either $\mu$ or $\tau$-flavor (red) which are degenerate in our Boltzmann scenario. The solid blue line is the degenerate FD equilibrium spectrum for any species of neutrino in the $\mathrm{SI} \nu$ scenario. The dotted black line at zero represents a nondegenerate spectrum at temperature $T_{\mathrm{cm}}$. In either scenario, there exists an overabundance of particles at high $\epsilon$. The positive slope of the SI $\nu$ curve corresponds to a higher temperature $T_{\nu}$ compared to $T_{\mathrm{cm}}$. We compare the energy scales $T_{\nu}$ and $T_{\mathrm{cm}}$ using the following definition

$$
\frac{T_{\nu}}{T_{\mathrm{cm}}}-1=a T_{\nu}-1 \equiv \Delta\left(a T_{\nu}\right)
$$


where we ascribe a unit conversion between scale factor and temperature such that the product is dimensionless and equal to unity when $T_{\nu}=10 \mathrm{MeV}$. At freeze-out (f.o.), we find

$$
\left.\Delta\left(a T_{\nu}\right)\right|_{\text {f.o. }}=2.463 \times 10^{-3},
$$

showing a slight increase in $T_{\nu}$ over $T_{\mathrm{cm}}$. Conversely, at low $\epsilon$ there exists a deficit of particles, which corresponds to a negative degeneracy parameter

$$
\left.\eta_{\nu}\right|_{\text {f.o. }}=-4.282 \times 10^{-3} \text {. }
$$

In the standard out-of-equilibrium scenario, the deficit arises from the up-scattering of low-energy neutrinos on higher-temperature charged leptons, given schematically in Eq. (3.8). The situation is similar for $\mathrm{SI} \nu$, as both elastic scattering and electron-positron annihilation [Eq. (3.9)] add energy and a modest number of particles to the neutrino seas. We can explain the behavior in Fig. 1 by examining Eqs. (3.5) and (3.6). The denominator in both equations is positive if $\eta_{\nu}=0$. If we solve the numerators for the energy added per particle we find

$$
\begin{gathered}
\frac{\partial \rho}{\partial n}>\frac{3 T_{\nu} n}{n_{\eta}} \Longrightarrow \frac{d T_{\nu}}{d t}>0 \\
\frac{\partial \rho}{\partial n}<\frac{4 \rho}{3 n} \Longrightarrow \frac{d \eta_{\nu}}{d t}>0
\end{gathered}
$$

where we ignore the redshift term for $d T_{\nu} / d t$ in Eq. (3.5). Assuming non-degenerate statistics, we calculate

$$
\begin{aligned}
\frac{3 T_{\nu} n}{n, \eta} & \simeq 3.288 T_{\nu}, \\
\frac{4 \rho}{3 n} & \simeq 4.202 T_{\nu} .
\end{aligned}
$$

Figure [4] in Ref. [31] shows that the weak-decoupling induced distortion in the neutrino seas is located at $\epsilon \simeq 5$ in the standard scenario. As this is larger than the two values above, the same energy per particle injection in the SI $\nu$ case would imply an increase in $T_{\nu}$ over $T_{\mathrm{cm}}$ and a decrease in $\eta_{\nu}$, which is borne out by Fig. 1.

We have given the values at freeze-out for $\eta_{\nu}$ and $T_{\mathrm{cm}} / T_{\nu}$. Figure 2 shows the evolution of $\Delta\left(a T_{\nu}\right)$ (solid blue line) and $\eta_{\nu}$ (dashed red line) from the initial values of zero to the freeze-out values as a function of $T_{\mathrm{cm}}$. The product $a T_{\nu}$ and the quantity $\eta_{\nu}$ can only change during weak decoupling, i.e., when the derivatives $\partial n /\left.\partial t\right|_{a}$ and $\partial \rho /\left.\partial t\right|_{a}$ are non-zero. Figure 2 illustrates the weak-decoupling epoch, starting at a few $\mathrm{MeV}$, and finishing slightly after $100 \mathrm{keV}$.

Neutrino self-interactions only indirectly influence the heat flow from the plasma into the neutrino seas. Figure 1 shows that the occupation numbers at freeze-out differ between the two scenarios. This is also the case during the entirety of weak decoupling. Modifying the occupation numbers and associated Pauli blocking factors will change the neutrino-chargedlepton scattering rates through the $f^{(k)}$ factors given in Eq. (2.10). The SI $\nu$ spectrum lies in between the two spectra for $e$-flavor and $\mu$-flavor in Fig. 1, implying a slight enhancement (suppression) for scattering between charged leptons and $e(\mu)$-flavor neutrinos. There is also an enhancement at low $\epsilon$ for any flavor for a substantially negative $\eta_{\nu}$. When calculating the radiation energy density at low temperatures, we find $N_{\text {eff }}=3.045$, a small overall increase of $10^{-3}$ compared to the result in Sec. 2.2. Although we evolve a non-zero degeneracy parameter, our result for $N_{\text {eff }}$ agrees with the $T_{\nu_{e}}=T_{\nu_{\mu, \tau}}$ model in the last row of Table [1] of Ref. [44]. 


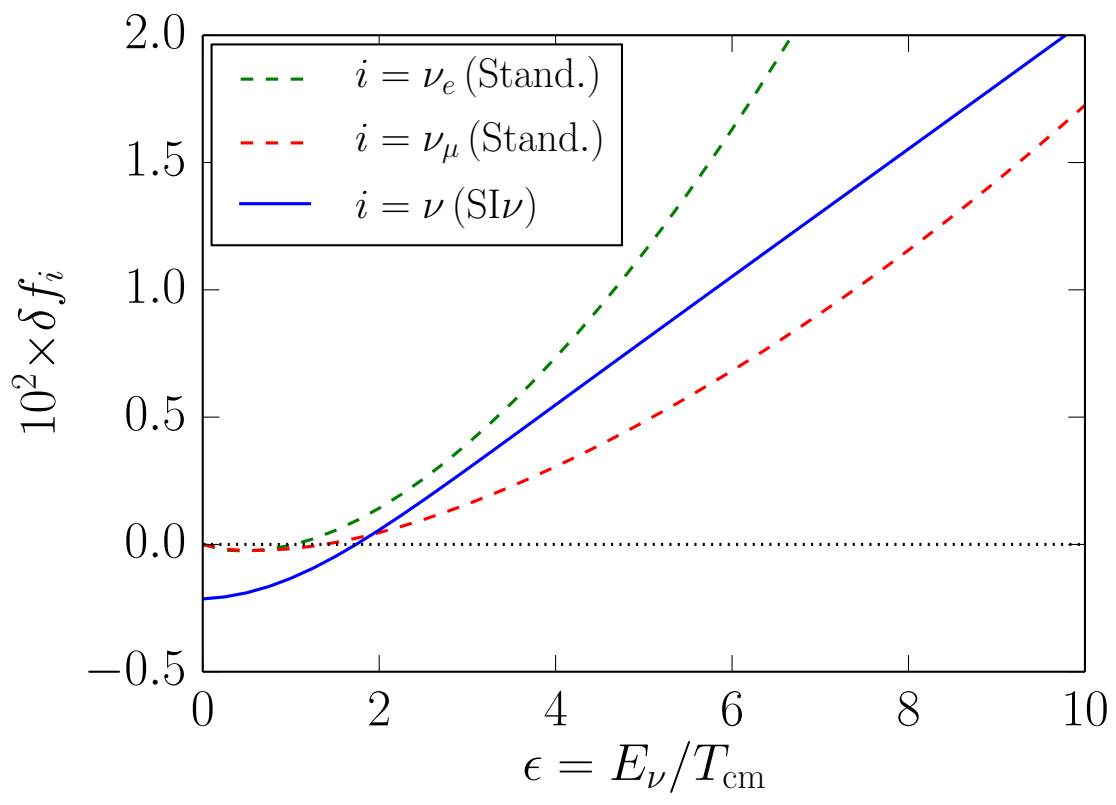

Figure 1. Freeze-out neutrino spectra in the standard scenario (dashed) and the SI $\nu$ scenario (solid) plotted against $\epsilon$. In the standard scenario of Boltzmann transport, the green dashed curve is the $e$ flavor and the red dashed curve is either $\mu$ or $\tau$. Vertical axis is the relative change to a non-degenerate FD spectrum in Eq. (3.14).

\subsubsection{Primordial Abundances}

The calculation for the primordial abundances is nearly identical in the standard and SI $\nu$ scenarios. The only difference between the two scenarios is in the neutron-to-proton rates. The SI $\nu$ scenario uses FD distributions with $T_{\nu}$ and $\eta_{\nu}$ compared to out-of-equilibrium occupation numbers $\left\{f_{\nu_{e}, \bar{\nu}_{e}}(\epsilon)\right\}$ for the standard scenario. The difference results in small relative changes for $Y_{\mathrm{P}}$ and $\mathrm{D} / \mathrm{H}$

$$
\begin{aligned}
& \delta Y_{\mathrm{P}} \simeq 4 \times 10^{-4}, \\
& \delta(\mathrm{D} / \mathrm{H}) \simeq 2 \times 10^{-4} .
\end{aligned}
$$

Both changes are well within observational uncertainties $[45,46]$. There exists a slight increase in the ratio ${ }^{7} \mathrm{Li} / \mathrm{H}$ with a relative change of $2 \times 10^{-4}$. The increase in all abundances is due to an increase in the neutron-to-proton ratio which results from a decrease in the rate $n\left(\nu_{e}, e^{-}\right) p$ as there exists fewer $\nu_{e}$ in the $\mathrm{SI} \nu$ case [47].

\section{Dark Radiation Addition}

\subsection{Dark Radiation Parameter}

This section and the succeeding one explore cosmological extensions to the $\mathrm{SI} \nu$ scenario. We begin by considering an addition of dark radiation [48] to the universal energy density. The dark radiation only interacts with the other components through gravitation. Within the 


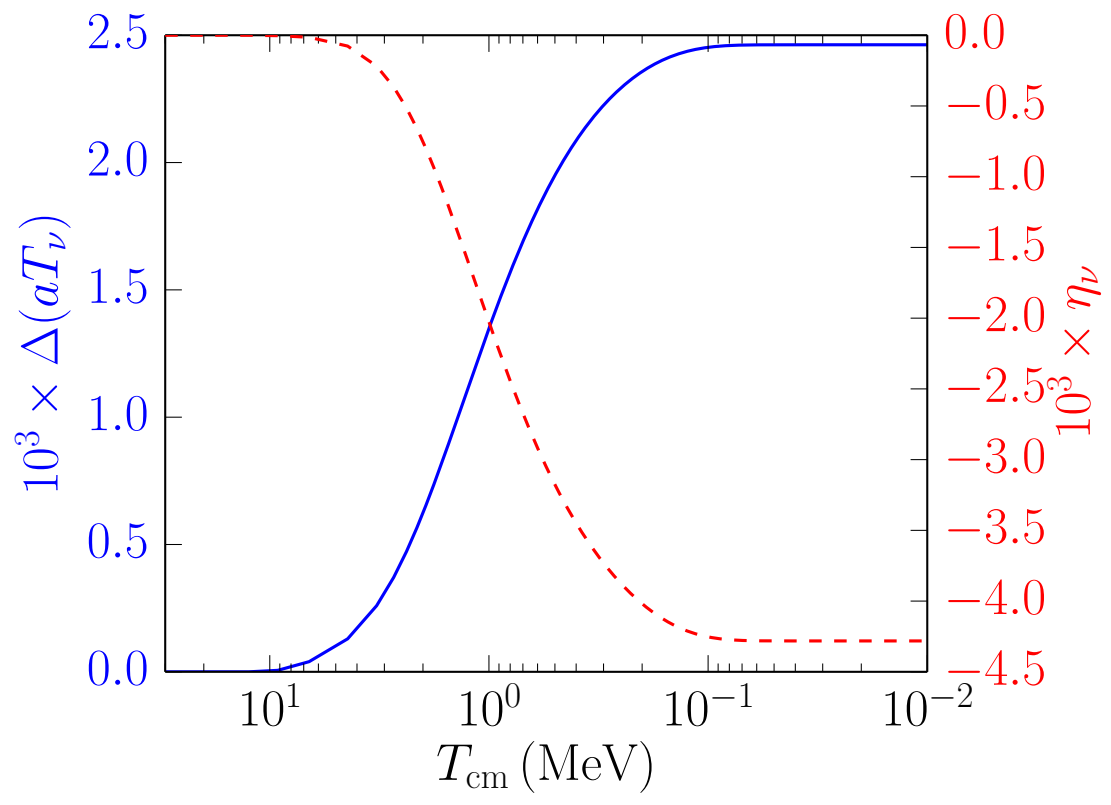

Figure 2. Evolution of $\Delta\left(a T_{\nu}\right)$ from Eq. (3.15) (solid blue curve) and $\eta_{\nu}$ (dashed red curve) plotted against $T_{\mathrm{cm}}$.

computation, only the Hubble expansion rate in Eq. (2.18) changes. We parameterize the dark radiation energy density by using the quantity $\delta_{\mathrm{dr}}$, introduced in [42]

$$
\rho_{\mathrm{dr}}=\frac{7 \pi^{2}}{120} T_{\mathrm{cm}}^{4} \delta_{\mathrm{dr}}
$$

After electron-positron annihilation terminates, we can calculate the change to $N_{\text {eff }}$ by adding $\rho_{\text {dr }}$ to $\rho_{\text {rad }}$ in Eq. (1.5)

$$
\Delta N_{\text {eff }}^{(\mathrm{dr})}=\left(\frac{11}{4}\right)^{4 / 3}\left(\frac{T_{\mathrm{cm}}}{T}\right)_{\text {f.o. }}^{4 / 3} \delta_{\mathrm{dr}} .
$$

Weak decoupling and QED corrections to the plasma equation of state modify the transfer of entropy from the charged leptons to the photons. The result is a freeze-out ratio $T_{\mathrm{cm}} / T$ larger than $(4 / 11)^{1 / 3}$, causing $\Delta N_{\mathrm{eff}}^{(\mathrm{dr})}$ to be larger than $\delta_{\mathrm{dr}}$. The increase is less than $1 \%$, so $\Delta N_{\mathrm{eff}}^{(\mathrm{dr})} \approx \delta_{\mathrm{dr}}$ to good approximation.

We do a parameter-space scan over $\delta_{\text {dr }}$ to calculate the changes to the neutrino spectra and primordial abundances in the $\mathrm{SI} \nu$ scenario.

\subsection{Results}

\subsubsection{Neutrino Spectra}

By construction, the dark radiation component has no direct effect on neutrino scattering and weak decoupling. When adding extra radiation energy density to the universe, we expect an earlier epoch of weak decoupling precipitated by the more rapid expansion rate. This 
conclusion is identical for either the standard or $\mathrm{SI} \nu$ scenarios. The final freeze-out spectra look similar to the blue curve in Fig. 1, and the evolution of $T_{\nu}$ and $\eta_{\nu}$ are qualitatively the same for the scenarios with and without dark radiation.

In Fig. 3 we plot four quantities related to $N_{\text {eff }}$ against the parameter $\delta_{\mathrm{dr}}$. The solid blue curve gives $\Delta N_{\text {eff }} \equiv N_{\text {eff }}-3$ and includes neutrino transport, QED corrections, and dark radiation in the $\mathrm{SI} \nu$ scenario. The dashed green curve is $\delta_{\mathrm{dr}}$ and close to the change in $N_{\text {eff }}$ from the addition of the dark radiation. The horizontal lines give the $1 \sigma$ uncertainty in $N_{\text {eff }}$ from Planck (dashed red line) [43] and a projection from Cosmic Microwave Background (CMB) Stage IV (dashed black line) [49].

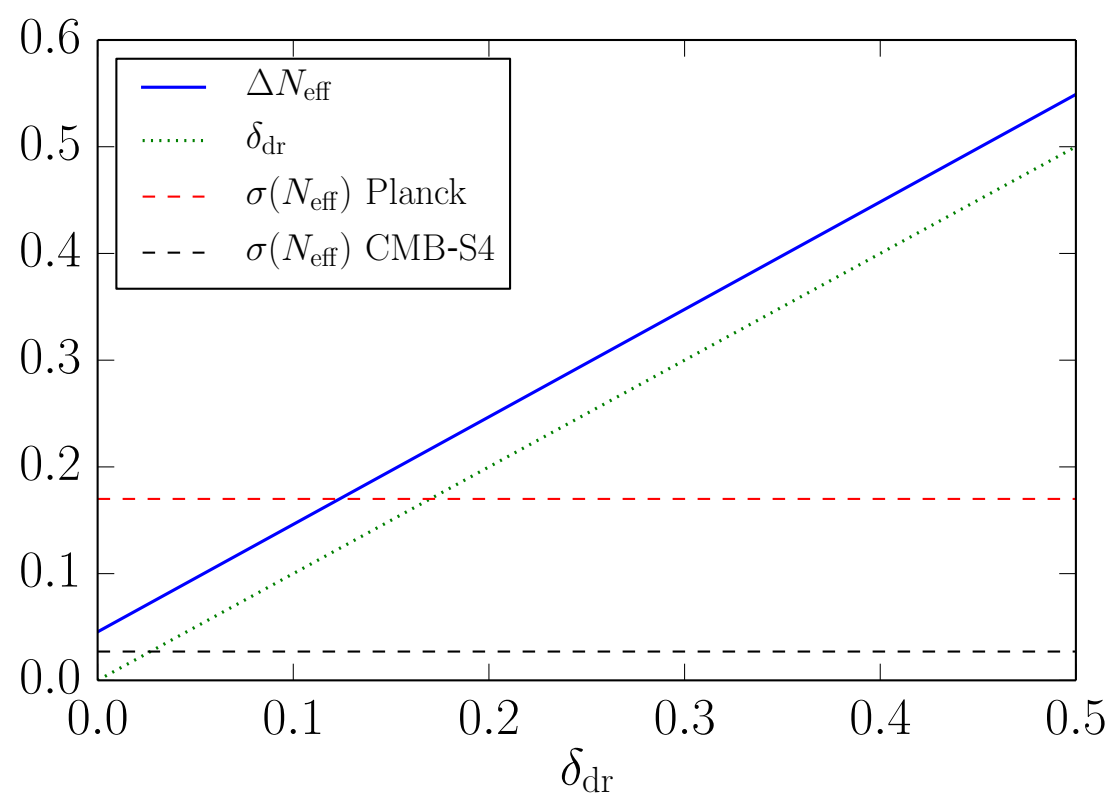

Figure 3. Change in $N_{\text {eff }}$ plotted against $\delta_{\mathrm{dr}}$.

The blue and green lines appear to run parallel to one another in Fig. 3, suggesting that changes to $N_{\text {eff }}$ from neutrino transport/QED corrections and dark radiation add linearly. This conclusion is indeed the case to good precision and is also true in the standard scenario. In models with dark radiation, the non-linear contribution to $\Delta N_{\text {eff }}$ comes from a change in the expansion rate which leads to an earlier epoch of weak decoupling and subsequently lowers the amount of heat flow from the plasma into the neutrino seas. This effect changes both the energy density in the neutrino seas and also the ratio $T_{\mathrm{cm}} /\left.T\right|_{\text {f.o. }}$ in Eq. (4.2). We have verified this behavior by comparing $N_{\text {eff }}\left(\delta_{\mathrm{dr}}=0.5\right)$ to $N_{\mathrm{eff}}\left(\delta_{\mathrm{dr}}=1.0\right)$ in the following manner

$$
2-\frac{N_{\mathrm{eff}}\left(\delta_{\mathrm{dr}}=1.0\right)-N_{\mathrm{eff}}\left(\delta_{\mathrm{dr}}=0\right)}{N_{\mathrm{eff}}\left(\delta_{\mathrm{dr}}=0.5\right)-N_{\mathrm{eff}}\left(\delta_{\mathrm{dr}}=0\right)} \simeq 9 \times 10^{-5}
$$

indicating that the degree of non-linearity is small and the blue and green curves in Fig. 3 are parallel to high precision. The non-linearity grows with increasing $\delta_{\mathrm{dr}}$ but is not a factor for modest values of $N_{\text {eff. }}$. We emphasize this analysis is for the $\mathrm{SI} \nu$ scenario but is directly applicable to the standard scenario. 


\subsubsection{Primordial Abundances}

Increasing $\delta_{\mathrm{dr}}$ precipitates an earlier epoch of weak freeze-out between the neutrinos and baryons, and results in a higher neutron-to-proton ratio during nuclear freeze-out. Both $Y_{\mathrm{P}}$ and $\mathrm{D} / \mathrm{H}$ increase in the presence of an over-abundance of neutrons. We direct the reader to Refs. [50, 51] for more information on extra radiation energy density in BBN, and in particular Ref. [52] for quantitative scaling forms of the abundances with $\Delta N_{\text {eff. }}$ Transport and self-interactions do little to modify the scaling forms and instead renormalize the values when $\delta_{\mathrm{dr}}=0$. We give specific values of $Y_{\mathrm{P}}$ and $\mathrm{D} / \mathrm{H}$ with relative changes from the standard baseline for the case $\delta_{\mathrm{dr}}=0.4009$

$$
\begin{array}{cccc}
Y_{\mathrm{P}}=0.2532 & \Longrightarrow & \delta Y_{\mathrm{P}}=2.161 \% & \left(\delta_{\mathrm{dr}}=0.4009\right), \\
\mathrm{D} / \mathrm{H}=2.769 \times 10^{-5} & \Longrightarrow \delta(\mathrm{D} / \mathrm{H})=5.385 \% & \left(\delta_{\mathrm{dr}}=0.4009\right) .
\end{array}
$$

We highlight that Eq. (4.4) shows that deuterium, not helium, has a larger relative change with the addition of dark radiation. In fact, this is a general trend in BBN and not specific to the value of $\delta_{\mathrm{dr}}$ taken in Eq. (4.4). Figure 10 of Ref. [52] shows the hypersensitivity of D/H to extra energy density. The reason for the result in Eq. (4.4) is the dark radiation precipitates an earlier freeze-out of nuclear reaction rates. Given the precision in measuring $\mathrm{D} / \mathrm{H}$, any $\mathrm{BBN}$ scenario which includes a dark radiation component must consider the impact on deuterium production.

\section{Lepton Asymmetric Initial Conditions}

We give a second example of a one-parameter extension to the $\mathrm{SI} \nu$ scenario by introducing an asymmetry between numbers of neutrinos and anti-neutrinos with the following quantity $[53]$

$$
L_{\nu_{i}}^{\star} \equiv \frac{n_{\nu_{i}}-n_{\bar{\nu}_{i}}}{\frac{2 \zeta(3)}{\pi^{2}} T_{\mathrm{cm}}^{3}}, \quad i=e, \mu, \tau
$$

where $\zeta(3)=1.202$ is the Riemann Zeta function of argument 3. The above definition is identical with the standard definition of lepton number

$$
L_{\nu_{i}}=\frac{n_{\nu_{i}}-n_{\bar{\nu}_{i}}}{n_{\gamma}}
$$

at high temperature where $T_{\mathrm{cm}}=T$. We use $L_{\nu_{i}}^{\star}$ as it is a comoving invariant throughout electron-positron annihilation and label it the comoving lepton number to distinguish it from Eq. (5.2). If self-interactions violate flavor conservation, than we would expect the comoving lepton number to be the same in all three flavors. Additionally, if self-interactions violate lepton number, we would expect $L_{\nu_{i}}^{\star}=0$ for all flavors at the commencement of BBN. We will consider the SI $\nu$ scenario of flavor-violation and lepton-number conservation in this section. Therefore, we only use a single quantity $L_{\nu}^{\star}$ to describe the asymmetry for any flavor.

\subsection{Differences in Implementation with Symmetric Scenario}

Neutrinos and anti-neutrinos will evolve uniquely in the $\mathrm{SI} \nu$ scenario with asymmetric initial conditions. In general, we need temperature and degeneracy quantities for the anti-neutrinos, namely, $T_{\bar{\nu}}$ and $\eta_{\bar{\nu}}$. The Lagrangian in Eq. (1.1) allows for $t$-channel scattering between neutrinos and anti-neutrinos, so the interaction

$$
\nu+\bar{\nu} \leftrightarrow \nu+\bar{\nu}
$$


holds the two seas in thermal equilibrium, implying $T_{\nu}=T_{\bar{\nu}}$. Equation (5.3), however, does not hold the two seas in chemical equilibrium. As a result, our model of SI $\nu$ with lepton degeneracy only requires the introduction of one new variable, namely $\eta_{\bar{\nu}}$. The set of variables $\left\{T_{\nu}, \eta_{\nu}, \eta_{\bar{\nu}}\right\}$ evolve in accordance with the definitions of energy and number density for neutrinos and anti-neutrinos. The expressions for $n_{\nu}\left(T_{\nu}, \eta_{\nu}\right)$ and $n_{\bar{\nu}}\left(T_{\nu}, \eta_{\bar{\nu}}\right)$ are identical to Eq. (3.2), except now $N_{\nu}=3$ for three flavors of neutrinos/anti-neutrinos. $\rho_{\nu}$ and $\rho_{\bar{\nu}}$ follow from analogy.

The asymmetry in the system implies there will be heat flow between the neutrinos and anti-neutrinos. Although the weak interaction can precipitate such a heat flow, the $t$ channel SI $\nu$ interaction will be much more efficient and will thermally equilibrate the two seas instantaneously. To account for this fact, we need to supplement the weak collision terms with an equilibration term $d \mathcal{E} / d t$

$$
\begin{aligned}
& \left.\frac{\partial \rho_{\nu}}{\partial t}\right|_{a}=\frac{T_{\nu}^{4}}{2 \pi^{2}} \sum_{i=1}^{3} \int d \epsilon \epsilon^{3}\left\{\mathcal{C}_{\nu e}\left[f^{(\mathrm{eq})}\left(\epsilon ; \eta_{\nu}\right)\right]+\mathcal{C}_{e^{-} e^{+}}\left[f^{(\mathrm{eq})}\left(\epsilon ; \eta_{\nu}\right)\right]\right\}+\frac{d \mathcal{E}}{d t} \\
& \left.\frac{\partial \rho_{\bar{\nu}}}{\partial t}\right|_{a}=\frac{T_{\nu}^{4}}{2 \pi^{2}} \sum_{i=1}^{3} \int d \epsilon \epsilon^{3}\left\{\mathcal{C}_{\nu e}\left[f^{(\mathrm{eq})}\left(\epsilon ; \eta_{\bar{\nu}}\right)\right]+\mathcal{C}_{e^{-} e^{+}}\left[f^{(\mathrm{eq})}\left(\epsilon ; \eta_{\bar{\nu}}\right)\right]\right\}-\frac{d \mathcal{E}}{d t} .
\end{aligned}
$$

The equilibration term is a weighted average of the energy and number flows due to the weak interaction. If we group the weak-collision terms into $d \rho_{\nu} /\left.d t\right|_{\mathrm{w}}$ and $d \rho_{\bar{\nu}} /\left.d t\right|_{\mathrm{w}}$, we find

$$
\begin{aligned}
\frac{d \mathcal{E}}{d t} & =\left[4 n_{\nu, \eta} n_{\bar{\nu}, \eta}\left(\rho_{\nu}+\rho_{\bar{\nu}}\right)-9 T_{\nu}\left(n_{\nu}^{2} n_{\bar{\nu}, \eta}+n_{\bar{\nu}}^{2} n_{\nu, \eta}\right)\right]^{-1} \\
& \times\left\{\left.3 T_{\nu}\left[4\left(n_{\nu} \rho_{\bar{\nu}} n_{\bar{\nu}, \eta}-n_{\bar{\nu}} \rho_{\nu} n_{\nu, \eta}\right)+9 T_{\nu} n_{\nu} n_{\bar{\nu}}\left(n_{\nu}-n_{\bar{\nu}}\right)\right] \frac{\partial n_{\nu}}{\partial t}\right|_{a}\right. \\
& \left.+4 n_{\nu, \eta} n_{\bar{\nu}, \eta}\left(\left.\rho_{\nu} \frac{\partial \rho_{\bar{\nu}}}{\partial t}\right|_{\mathrm{w}}-\left.\rho_{\nu} \frac{\partial \rho_{\bar{\nu}}}{\partial t}\right|_{\mathrm{w}}\right)+9 T_{\nu}\left(\left.n_{\bar{\nu}}^{2} n_{\nu, \eta} \frac{\partial \rho_{\nu}}{\partial t}\right|_{\mathrm{w}}-\left.n_{\nu}^{2} n_{\bar{\nu}, \eta} \frac{\partial \rho_{\bar{\nu}}}{\partial t}\right|_{\mathrm{w}}\right)\right\}
\end{aligned}
$$

In writing Eq. (5.6), we have set $\partial n_{\bar{\nu}} /\left.\partial t\right|_{a}=\partial n_{\nu} /\left.\partial t\right|_{a}$ as the weak-collision terms conserve lepton number. Those number-flow terms are analogous to Eq. (3.10). The derivatives for $T_{\nu}, \eta_{\nu}$, and $\eta_{\bar{\nu}}$ follow from Eqs. (3.5) and (3.6).

Initially at high temperature, neutrinos and anti-neutrinos are in thermal equilibrium with the plasma and $T_{\nu}=T$. In addition, the weak interaction ensures neutrinos and antineutrinos are in chemical equilibrium and have degeneracy parameters equal in magnitude and opposite in sign. The equation

$$
L_{\nu}^{\star}=\frac{1}{12 \zeta(3)}\left(\pi^{2} \xi+\xi^{3}\right)
$$

relates the comoving lepton number to the degeneracy parameter [23]. We begin the calculation by setting $\eta_{\nu}=\xi$ and $\eta_{\bar{\nu}}=-\xi$.

\subsection{Results}

\subsubsection{Neutrino Spectra}

We give an example of an asymmetric SI $\nu$ calculation with $L_{\nu}^{\star}=2.897 \times 10^{-3}$ in Fig. 4 . The two curves of Fig. 4 show the evolution of the degeneracy parameters for neutrinos (solid blue curve) and anti-neutrinos (solid red curve) as a function of $T_{\mathrm{cm}}$. These two curves evolve 
similarly albeit with a noticeable offset due to the initial asymmetry. Figure 4 clearly shows the composite neutrino/anti-neutrino system is not in equilibrium as $\eta_{\nu} \neq-\eta_{\bar{\nu}}$ once weak decoupling begins. We do not show the evolution of $T_{\nu}$ as its behavior is similar to that of $T_{\nu}$ in the lepton-symmetric case in Fig. 2. The paucity of anti-neutrinos leads to a suppression of the Pauli blocking factors in the weak collision terms, and therefore an increase in the scattering rates of charged leptons with anti-neutrinos. Conversely, the overabundance of neutrinos enhances the Pauli blocking factors and decreases those scattering rates. The two effects together imply $d \mathcal{E} / d t>0$. Overall, the increase in the anti-neutrino scattering rates and the decrease in the neutrino rates nearly cancel and the end result is the evolution of $T_{\nu}$ in the lepton-asymmetric case differs only slightly from the lepton-symmetric case. The lepton-asymmetric case does induce a larger heat flow than the lepton-symmetric case, but the difference is below the resolution of the plot for $T_{\nu}$ in Fig. 2.

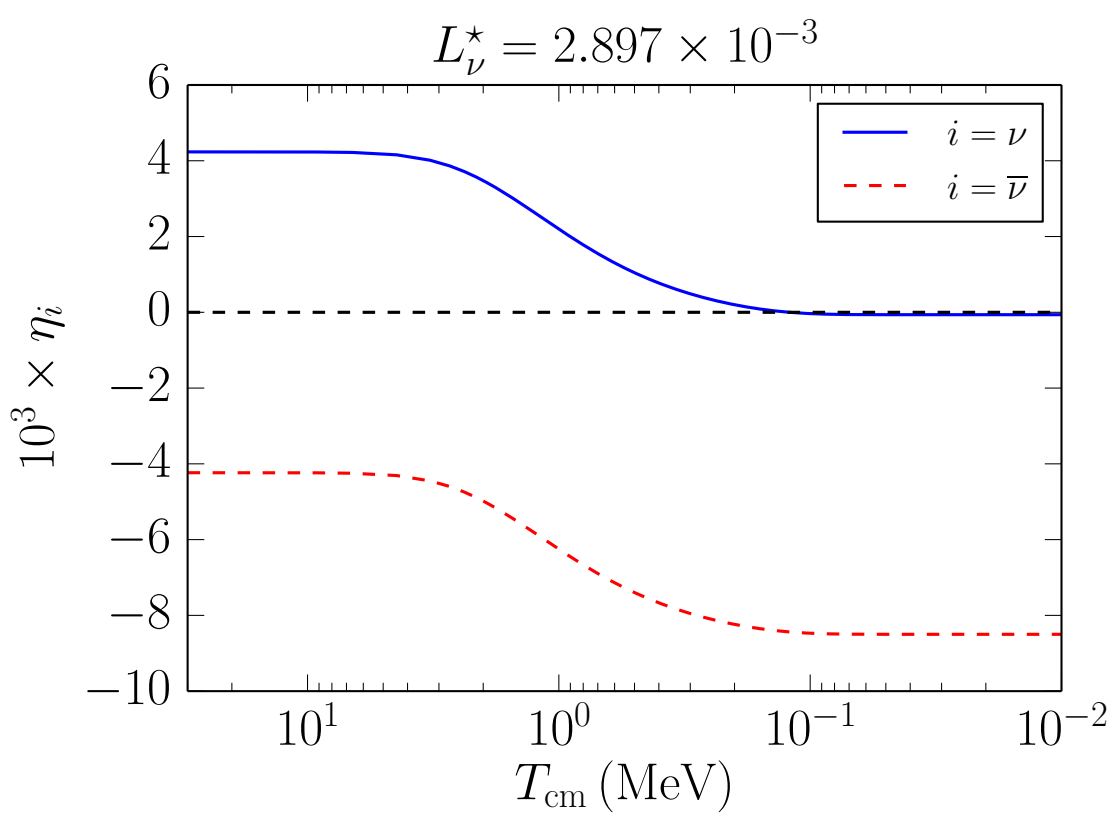

Figure 4. Evolution of $\eta_{i}, i=\nu, \bar{\nu}$ as a function of $T_{\mathrm{cm}}$. The comoving lepton number is $L_{\nu}^{\star}=$ $2.897 \times 10^{-3}$.

We have shown results for the neutrino parameters in the case $L_{\nu}^{\star}>0$. If $L_{\nu}^{\star}<0$, then we are in the opposite $C P$ system and the results corresponding to Fig. 4 would be $C P$ conjugates. The quantity $N_{\text {eff }}$ does change in an asymmetric scenario as this system has a larger energy density. However, $N_{\text {eff }}$ only has sensitivity to the magnitude of $L_{\nu}^{\star}$. Both the larger energy density from degenerate FD statistics and neutrino energy transport are symmetrical for $\pm L_{\nu}^{\star}$. Therefore, $N_{\text {eff }}$ is immune to the sign of $L_{\nu}^{\star}$.

\subsubsection{Primordial Abundances}

As opposed to $N_{\text {eff }}$, the primordial abundances are indeed sensitive to the sign of $L_{\nu}^{\star}$. The neutrino spectra are inputs into the neutron-to-proton rates and therefore change the evolution of the neutron-to-proton ratio. We execute a parameter space scan over $L_{\nu}^{\star}$ to examine 
how the lepton asymmetry affects the primordial abundances in the $\mathrm{SI} \nu$ scenario. Figure 5 shows $Y_{\mathrm{P}}$ as a function of $\left|L_{\nu}^{\star}\right|$, where the blue curve gives $Y_{\mathrm{P}}$ for $L_{\nu}^{\star}>0$ and the red curve gives $Y_{\mathrm{P}}$ for $L_{\nu}^{\star}<0$. We only show $Y_{\mathrm{P}}$ in Fig. 5 , but the other abundances have similar shapes as seen in Fig. [14] in Ref. [53]. In particular, D/H follows qualitatively the same shape as the curves in Fig. 5 and has a relative change of $\sim 1 / 2$ as compared to $Y_{\mathrm{P}}$ at all values of $L_{\nu}^{\star}$.

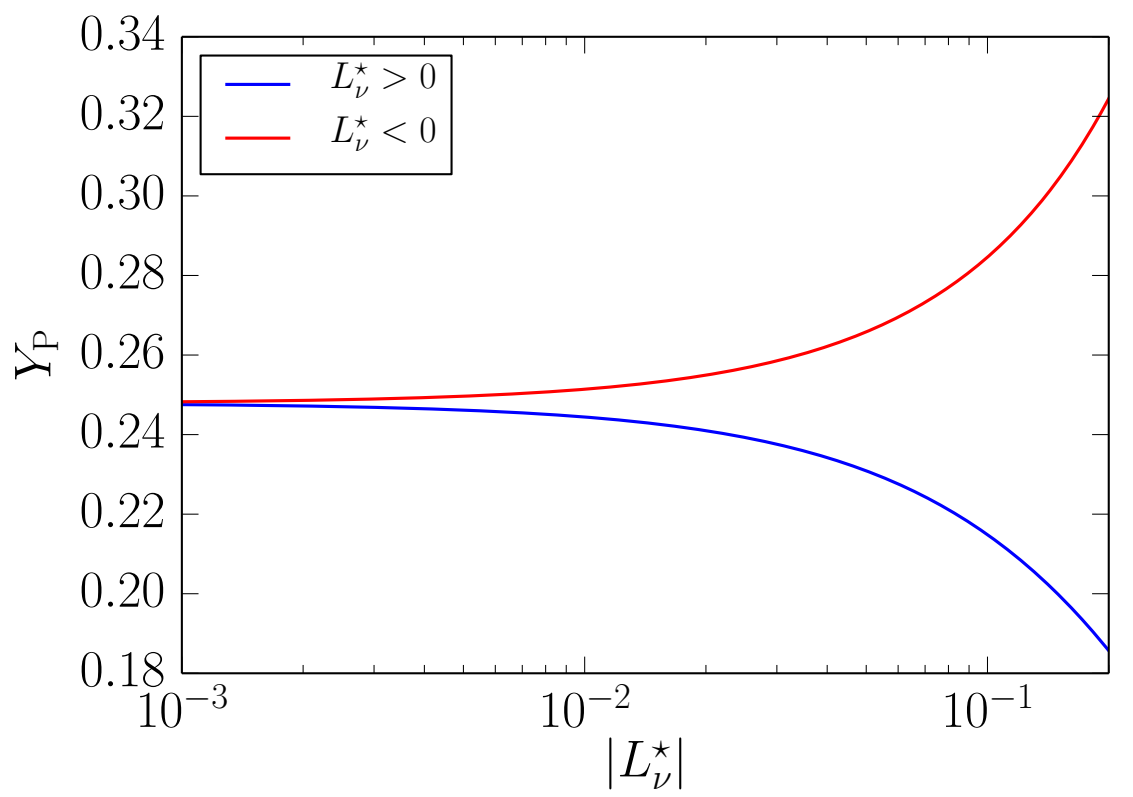

Figure 5. $\quad Y_{\mathrm{P}}$ plotted against $\left|L_{\nu}^{\star}\right|[$ Eq. (5.1)] in the $\mathrm{SI} \nu$ scenario.

Before we conclude, we provide an example model with specific values. If we desire to decrease the helium mass fraction by $10 \%$, we would need a positive comoving lepton number of $L_{\nu}^{\star} \simeq 0.073$. A $10 \%$ change in $Y_{\mathrm{P}}$ would be difficult to reconcile with current observational bounds [45]. The extra energy density from this value of comoving lepton number translates to $\Delta N_{\text {eff }}=0.06$, which is consistent with Ref. [43]. We conclude that $Y_{\mathrm{P}}$ has the potential to be a better probe for $L_{\nu}^{\star}$ than $N_{\text {eff. }}$ Our conclusion is for the $\mathrm{SI} \nu$ scenario and is directly applicable to the standard scenario extension with lepton asymmetry.

\section{Conclusions}

\subsection{Summary}

Self-interacting neutrinos constitute a model of Beyond Standard Model (BSM) physics with implications for BBN. Neutrinos go out of thermal and chemical equilibrium during BBN in the standard scenario. Figure 1 shows that these deviations from equilibrium are small, although potentially detectable in future measurements of $N_{\text {eff }}$ [49]. Self-interacting neutrinos have little influence on the magnitude of the deviations, and instead reshuffle the neutrino energy among the flavors while preserving the general shape of the spectra. Figure 2 shows 
that self-interactions modify the spectral parameters by a few parts in $10^{3}$. Although the physics governing neutrino interactions is different, the standard and $\mathrm{SI} \nu$ scenarios do not predict any substantial differences for cosmological observables/parameters at present or future precision [see Eqs. (3.22) and (3.23)].

We have extended our BSM model to include self-interactions and dark radiation. With the inclusion of extra radiation energy density, weak decoupling terminates at an earlier epoch and less heat flows from the electromagnetic plasma to the neutrino seas. Equation (4.3) shows the change in neutrino energy density caused by the earlier freeze-out is small. As a result, self-interactions perturb the neutrino seas in much the same manner as they did without the addition of the dark radiation. Figure 3 shows how $N_{\text {eff }}$ changes with the addition of dark radiation, which is nearly an incoherent sum of the extra energy density and the effects from weak decoupling. Although Fig. 3 is for the $\mathrm{SI} \nu$ scenario, $N_{\text {eff }}$ behaves in quantitatively the same manner for the one-parameter extension to the standard scenario.

Finally, we extended the SI $\nu$ scenario to include lepton asymmetry. In the class of models we explore, the self-interactions always maintain flavor-identical distributions among the neutrino and anti-neutrino seas. The flavor-independence of the self-interactions results in the lepton numbers necessarily being identical to one another for the three flavors. The difference between this scenario and the ones with symmetric initial conditions is that the neutrino and anti-neutrino components can have different spectra. Figure 4 shows different evolutions for the degeneracy parameters in a SI $\nu$ scenario where $L_{\nu}^{\star}=2.897 \times 10^{-3}$. Although the degeneracy parameters undergo different evolutions compared to the symmetric scenario, the net change from the initial starting point is still a few parts in $10^{3}$. As a result, predictions on cosmological parameters/observables mainly scale with the presence of a non-zero $L_{\nu}^{\star}$, and self-interactions produce higher-order effects. Figure 5 gives the primordial mass fraction of ${ }^{4} \mathrm{He}$ as a function of $L_{\nu}^{\star}$ in the $\mathrm{SI} \nu$ scenario. Lepton degeneracy sources an increasing (decreasing) $Y_{\mathrm{P}}$ with decreasing (increasing) $L_{\nu}^{\star}$ while self-interactions simply translate the curves to slightly higher values.

\subsection{Discussion}

The motivation for this work was to determine the dynamics of weak decoupling and primordial nucleosynthesis in the presence of SI $\nu$. Our impetus for this decision is two-fold. First, the neutrinos experience kinetic evolution during this epoch, as scattering rates are neither large enough to maintain equilibrium nor small enough to preclude thermal contact. If new neutrino physics manifests during BBN, standard-model physics will not be able to erase these signals. Second, cosmological parameters/observables directly influenced by the physics of the BBN epoch have already been measured. Advances in precision on $\left\{N_{\text {eff }}, Y_{\mathrm{P}}, \mathrm{D} / \mathrm{H}\right\}$ will either place stronger constraints or reveal signatures of new physics. Coupled together, the kinetic regime and the ability to make measurements which provide diagnostics of the physics operating during this epoch provide researchers with a tool to explore new physical theories.

Our intent for this work was to quantify how self-interactions, and solely self-interactions, modify the neutrino spectra and concomitant light-element synthesis. Section 3 presents the results for the $\mathrm{SI} \nu$ scenario which amount to less than a $1 \%$ change from the standard BBN scenario. The same level of relative changes between the standard and $\mathrm{SI} \nu$ scenarios occur when we add dark radiation or lepton asymmetry to BBN. We conclude that BBN does not provide any additional constraints on a scenario with only self-interacting neutrinos.

We chose a limited class of SI $\nu$ models for this work. Namely, we operate in the following $\mathrm{SI} \nu$ framework: (1) the interaction matrix, $g_{i j}$ in Eq. (1.2), is agnostic to any particular flavor; 
(2) the self-interactions are $C P$-symmetric; (3) $\varphi$ is a real scalar; (4) the mass of $\varphi$ is large compared to BBN energy scales; and finally (5), the neutrinos stay self-coupled throughout BBN. We contemplate the repercussions if we relax each one of these limitations in turn.

Our choice for $g_{i j}$ forced all flavors of neutrinos to have the same distribution. Had we set the off-diagonal terms of $g_{i j}$ to zero, then we would have needed separate parameters $T_{\nu_{i}}$ and $\eta_{\nu_{i}}$ for each flavor. Given how little self-interactions changes cosmological parameters/observables, we do not expect this class of models to alter our conclusions significantly. Table [1] in Ref. [44] appears to bear out this conclusion for $N_{\text {eff. }}$ Moreover, had we introduced a flavor asymmetry in the interaction matrix, i.e., $g_{e e} \neq g_{\mu \mu}$, then we might expect some flavors of neutrinos to stay in self-equilibrium and others to evolve in an out-of-equilibrium fashion. Figure 1 shows there is little difference between these two kinds of distributions, so again we expect little effect on parameters. What may be different would be the case where there exists lepton asymmetries. If the self-interacting Lagrangian is indeed flavor biased, then lepton numbers can be different for different flavors, and oscillations would play a dominant effect during BBN [54-56]. Furthermore, the flavor-asymmetric scenario could have severe repercussions on neutrino free-streaming during atomic recombination, if the neutrino gas is only partially self-coupled. Such a scenario requires a calculation with vacuum neutrino oscillations, but we can give a qualitative description here. In the standard cosmology, free-streaming neutrinos cause a phase shift in the CMB acoustic peaks $[57,58]$. The SI $\nu$ gas eliminates that phase shift and utilizes extra dark radiation to remedy this particular temperature power-spectrum defect. If not all the neutrinos were to be self-coupled, then the free-streaming neutrino component would induce a small phase shift, and the amount of dark radiation would necessarily be smaller. At the current epoch, the effect would be to increase the Hubble parameter only slightly.

In addition to all flavors being self-interacting, SI $\nu$ scenarios which remedy the Hubble parameter tension also stipulate the anti-neutrinos to be self-interacting. If the interacting Lagrangian in Eq. (1.1) is not $C P$-symmetric, then models which use $\mathrm{SI} \nu$ to resolve cosmological tensions will also confront the same problems as the flavor-asymmetric models discussed above. From the perspective of BBN, if anti-neutrinos do not remain in a self-equilibrium, then the anti-neutrino spectra would follow the dashed lines in Fig. 1 while the neutrinos followed the solid line. Again, we do not expect the asymmetry to cause significant changes in cosmological parameters related to BBN. With the introduction of a lepton number, we still would expect equal lepton numbers in all flavors as self-interactions will change the flavor content in the neutrino sector and standard model interactions will then equilibrate the numbers of neutrinos and anti-neutrinos such that all have identical chemical potentials.

In our analyses of neutrino-anti-neutrino asymmetry, we have always made the assumption that the net lepton number (i.e., the sum of the three flavor lepton numbers) cannot change. For the Lagrangian in Eq. (1.1), this is certainly true. We could alter the Lagrangian to include right-handed neutrinos, with an operator such as $\overline{\nu_{R}} \nu_{L} \varphi$. For Majorana character, left-handed states could undergo spin-flips to the right-handed states at the same rate as the processes which equilibrate the neutrino/anti-neutrino seas. A lepton asymmetry cannot persist in such a scenario. If neutrinos are Dirac fermions, then the spin-flip populates the opposite helicity states. These states are inactive under the weak interaction, but would still have ramifications on $\mathrm{BBN}$ as they contribute to the radiation energy density and would raise $N_{\text {eff. }}$ The spin-flip interactions would have to freeze-out well before BBN [59] for a $\mathrm{SI} \nu$ model with right-handed neutrinos to be viable. As a corollary, BBN would proceed without $\mathrm{SI} \nu$ interactions but with the addition of dark radiation. Alternatively, if we were to 
consider vector mediators, then lepton number would not be conserved and we would expect a symmetric system. We do not anticipate any further implications for either BBN or photon decoupling if the mediator is a vector. Another possibility in the class of SI $\nu$ models is for a pseudoscalar mediator. In this scenario, neutrinos may recouple after weak decoupling [60]. Some late portion of recombination could transpire with equilibrium neutrino spectra, but BBN would occur with out-of-equilibrium distributions identical to the standard scenario.

Returning to the scalar-mediator scenario, we only considered the case when the mass of the mediator is much larger than BBN energy scales. If this were not the case, then it is possible for the mediator to be produced on shell, through reactions like $\nu+\nu \rightarrow \phi+\phi$, during BBN. As we take the self-interactions strong enough to ensure neutrino self-equilibrium throughout BBN, light mediators will continue to remain in equilibrium with the neutrino seas until the neutrinos decouple from themselves at late times. There are three ramifications of this. First, the $\varphi$-sea acts as a heat bath for neutrinos without conserving number. With a heat bath and strong coupling, the neutrino seas are in thermal and chemical equilibrium with temperature $T_{\nu}$ and vanishing $\eta_{\nu}$. The evolution of $T_{\nu}$ and $\eta_{\nu}$ in Eqs. (3.5) and (3.6) no longer apply, and we would have to construct an analog of Eq. (2.9) for the $\nu$ - $\varphi$ system. Second, if a $\varphi$-sea persists to times after weak decoupling, then there will be extra energy density in the $\nu-\varphi$ system which will become only neutrino and therefore increase the radiation energy density and $N_{\text {eff. }}$. This extra energy density is not dark radiation and will have ramifications on temperature power spectra [22]. Lastly, the energy in the $\varphi$-sea will heat the neutrinos to higher temperatures. If this occurs prior to helium formation, the higher neutrino temperature will cause a lower neutron-to-proton ratio and a smaller amount of helium.

Our final retrospection on the $\mathrm{SI} \nu$ framework presented in this paper, is if we remove the stipulation that the neutrinos stayed in self-equilibrium throughout BBN. This is a class of models where $G_{\text {eff }} \sim G_{F}$ and would not be able to alleviate the Hubble tension. As the self-interactions only reshuffle neutrino energy density, we would expect changes to BBN observables to be somewhere between the standard and full SI $\nu$ scenarios. In this case, we would not expect significant changes in $\left\{N_{\text {eff }}, Y_{\mathrm{P}}, \mathrm{D} / \mathrm{H}\right\}$. The more interesting scenario would be for the case of mediators with masses low enough to have non-zero densities during $\mathrm{BBN}$. If the self-interactions freeze-out prior to or during BBN, the population of $\varphi$ may still be present and decay into high-energy neutrinos. Such a decay is necessarily an out-ofequilibrium process and would require a transport calculation. If this decay occurs during weak decoupling, there exists the possibility that energy and entropy flow from the neutrino seas into the electromagnetic plasma, opposite of the direction in standard weak decoupling. The abundances, especially $\mathrm{D} / \mathrm{H}$, are sensitive to this entropy flow. Given the precision on measuring $\mathrm{D} / \mathrm{H}, \mathrm{BBN}$ has the potential of putting stricter limits for the "weak-selfinteracting" case than the stronger case considered in this work.

There can be other, non-cosmology constraints on some self-interacting sterile neutrino models. Such models with low enough scalar mass $m_{\varphi}$ and utilizing Majorana neutrinos can produce a unique neutrino-less double beta decay signature. This is where the double beta decay nuclear mass difference is shared among two electrons and an undetected scalar [61]. This distinctive phase space signature has allowed Majoron-like models [17-19] with effective couplings $g>10^{-3}$ [62] to be constrained. Future tonne-scale double beta decay detectors will have higher sensitivity and therefore likely will be able to extend these constraints. Finally, the $Z^{0}$-width measurement obviously provides a stringent limit on the triplet versions but not the singlet versions of the Majoron-like models discussed above. There can be other such 
constraints, for example on four-neutrino couplings [63].

Neutrino physics can play a significant, even dominant role in the dynamics, evolution, and nucleosynthesis in some compact object environments. These include, for example, core collapse supernovae and binary neutron star mergers. Not surprisingly, BSM neutrino selfinteractions may have dramatic effects in these venues. These effects could lead to constraints which can complement those derived from the early universe considerations discussed in this work. However, the origin of some of the potentially constrainable compact object effects is rooted in the same self-equilibration and flavor changing physics that affects weak decoupling and BBN in the early universe. When it comes to neutrino self-interactions and flavor, the key difference between compact objects and the early universe is the very low entropy per baryon in the former venue. For example, the collapsing cores of supernovae are characterized by low entropy and highly degenerate electron lepton number carried by seas of electrons and electron neutrinos. When the neutrinos become trapped by coherent neutral current scattering on heavy nuclei, self-interactions can do two things: (1) effect rapid self-equilibration among the neutrinos, very much like the self-equilibration in the early universe discussed above; and (2) rapidly re-distribute the degenerate electron lepton number among all three neutrino flavors. Electron neutrinos can be converted to $\mu$ and $\tau$ species, opening phase space for electron capture, $e^{-}+p \rightarrow n+\nu_{e}$. Very quickly the electron lepton number residing in the degenerate sea of electrons is redistributed and entropy is generated. This can result in dramatic alteration of the physics of collapse [3, 64]. Moreover, after core bounce, the neutrinos can be "cooled" by adiabatic expansion of the strongly self-coupled neutrino gas. This could lead to a constraint if neutrino energies are reduced below the thresholds for the IMB [65] and Kamiokande II [66] experiments which detected the neutrino burst from supernova SN1987a [67].

In closing, we mention the importance of deuterium in BBN constraints. Although the topic of this work is $\mathrm{SI} \nu$, our analysis applies to other BSM scenarios. Cosmological models which posit extra radiation energy density during BBN [68-71] necessarily change the abundances. In particular, helium and deuterium increase if that energy density is dark radiation, i.e., $\delta_{\mathrm{dr}}>0$. This can be remedied using a positive lepton number, as both $Y_{\mathrm{P}}$ and $\mathrm{D} / \mathrm{H}$ decrease, while $N_{\text {eff }}$ and other neutrino cosmology parameters (i.e., the sum of the light neutrino masses) are little changed by modest values of $L_{\nu}^{\star}\left(N_{\text {eff }}\right.$ and the abundances scale differently for large values of $L_{\nu}^{\star}$ [72]). However, the results of Secs. 4 and 5 show that the two abundances do not have identical scaling relations if $\delta_{\mathrm{dr}}$ and $L_{\nu}^{\star}$ are to be used in concert. In other words, dark radiation and lepton asymmetry cannot together assuage tensions in abundances. Given the precision in measuring the primordial $\mathrm{D} / \mathrm{H}$ observable, changes of even a few percent could cause a strong tension. The situation would be even worse with precise measurements of ${ }^{3} \mathrm{He}$ and ${ }^{7} \mathrm{Li}$, as the sign of the relative abundance changes is different compared to $Y_{\mathrm{P}}$ and $\mathrm{D} / \mathrm{H}$. At this point, other physical mechanisms would need to be invoked to mitigate internal $\mathrm{BBN}$ inconsistencies. Returning to the $\mathrm{SI} \nu$ scenario, picking a mass of the $\varphi$ mediator for neutrino entropy creation during $\mathrm{BBN}$ could change $Y_{\mathrm{P}}$ and $\mathrm{D} / \mathrm{H}$ such that the two scaling relationships are identical when dark radiation is included. Such a requirement on $\mathrm{SI} \nu$ - or any other BSM scenario which must reconcile $Y_{\mathrm{P}}$ and $\mathrm{D} / \mathrm{H}$ with $N_{\text {eff }}$ - will narrowly constrict the available parameter space. 


\section{Acknowledgments}

We thank Luke Johns, Amol Patwardhan, André de Gouvêa, Yue Zhang, Walter Tangarife, and Julien Froustey for useful discussions. This research was conducted through the Network for Neutrinos, Nuclear Astrophysics and Symmetries collaboration and is supported by National Science Foundation, grant PHY-1630782, and Heising-Simons Foundation, grant 2017-228. We also acknowledge NSF grants PHY-1614864 and PHY-1914242 at University of California San Diego. This research used resources provided by the Los Alamos National Laboratory Institutional Computing Program, which is supported by the U.S. Department of Energy National Nuclear Security Administration under Contract No. 89233218CNA000001.

\section{References}

[1] M. Tanabashi and Particle Data Group, Review of Particle Physics*, Phys. Rev. D 98 (Aug, 2018) 030001.

[2] G. Raffelt and J. Silk, Light neutrinos as cold dark matter, Physics Letters B 192 (Jun, 1987) 65-70.

[3] G. M. Fuller, R. Mayle, and J. R. Wilson, The Majoron Model and Stellar Collapse, ApJ 332 (Sep, 1988) 826.

[4] B. Dasgupta and J. Kopp, Cosmologically Safe eV-Scale Sterile Neutrinos and Improved Dark Matter Structure, Phys. Rev. Lett. 112 (Jan, 2014) 031803, [arXiv:1310.6337].

[5] J. F. Cherry, A. Friedland, and I. M. Shoemaker, Neutrino Portal Dark Matter: From Dwarf Galaxies to IceCube, ArXiv e-prints (Nov., 2014) [arXiv:1411.1071].

[6] N. Saviano, A. Mirizzi, O. Pisanti, P. D. Serpico, G. Mangano, and G. Miele, Multimomentum and multiflavor active-sterile neutrino oscillations in the early universe: Role of neutrino asymmetries and effects on nucleosynthesis, Phys. Rev. D 87 (Apr., 2013) 073006, [arXiv: 1302.1200].

[7] F. Forastieri, M. Lattanzi, G. Mangano, A. Mirizzi, P. Natoli, and N. Saviano, Cosmic microwave background constraints on secret interactions among sterile neutrinos, Journal of Cosmology and Astro-Particle Physics 2017 (July, 2017) 038.

[8] L. Johns and G. M. Fuller, Self-interacting sterile neutrino dark matter: The heavy-mediator case, Phys. Rev. D 100 (Jul, 2019) 023533, [arXiv:1903.0829].

[9] A. de Gouvêa, M. Sen, W. Tangarife, and Y. Zhang, The Dodelson-Widrow Mechanism In the Presence of Self-Interacting Neutrinos, arXiv e-prints (Oct, 2019) arXiv:1910.04901, [arXiv: 1910.0490$]$.

[10] F.-Y. Cyr-Racine and K. Sigurdson, Limits on Neutrino-Neutrino Scattering in the Early Universe, Phys. Rev. D90 (2014), no. 12 123533, [arXiv:1306.1536].

[11] C. D. Kreisch, F.-Y. Cyr-Racine, and O. Doré, The Neutrino Puzzle: Anomalies, Interactions, and Cosmological Tensions, arXiv e-prints (Feb, 2019) arXiv:1902.00534, [arXiv: 1902.0053].

[12] G. Barenboim, P. B. Denton, and I. M. Oldengott, Constraints on inflation with an extended neutrino sector, Phys. Rev. D99 (2019), no. 8 083515, [arXiv:1903.0203].

[13] K. S. Babu, G. Chauhan, and P. S. Bhupal Dev, Neutrino Non-Standard Interactions via Light Scalars in Earth, Sun, Supernovae and Early Universe, arXiv e-prints (Dec., 2019) arXiv:1912.13488, [arXiv: 1912.1348].

[14] M. Blennow, A. Mirizzi, and P. D. Serpico, Nonstandard neutrino-neutrino refractive effects in dense neutrino gases, Phys. Rev. D78 (2008) 113004, [arXiv:0810.2297]. 
[15] A. Das, A. Dighe, and M. Sen, New effects of non-standard self-interactions of neutrinos in a supernova, JCAP 1705 (2017), no. 05 051, [arXiv:1705.0046].

[16] A. Dighe and M. Sen, Nonstandard neutrino self-interactions in a supernova and fast flavor conversions, Phys. Rev. D97 (2018), no. 4 043011, [arXiv:1709.0685].

[17] G. B. Gelmini and M. Roncadelli, Left-handed neutrino mass scale and spontaneously broken lepton number, Physics Letters B 99 (Mar, 1981) 411-415.

[18] H. M. Georgi, S. L. Glashow, and S. Nussinov, Unconventional model of neutrino masses, Nuclear Physics B 193 (Dec, 1981) 297-316.

[19] B. Grinstein, J. Preskill, and M. B. Wise, Neutrino masses and family symmetry, Physics Letters B 159 (Sep, 1985) 57-61.

[20] J. M. Berryman, A. De Gouva, K. J. Kelly, and Y. Zhang, Lepton-Number-Charged Scalars and Neutrino Beamstrahlung, Phys. Rev. D97 (2018), no. 7 075030, [arXiv:1802.0000].

[21] K. J. Kelly and Y. Zhang, Mononeutrino at DUNE: New Signals from Neutrinophilic Thermal Dark Matter, Phys. Rev. D99 (2019), no. 5 055034, [arXiv:1901.0125].

[22] N. Blinov, K. J. Kelly, G. Krnjaic, and S. D. McDermott, Constraining the Self-Interacting Neutrino Interpretation of the Hubble Tension, Phys. Rev. Lett. 123 (Nov, 2019) 191102, [arXiv: 1905.0272].

[23] E. W. Kolb and M. S. Turner, The Early Universe. Addison-Wesley Publishing Co., 1990.

[24] A. G. Riess, S. Casertano, W. Yuan, L. M. Macri, and D. Scolnic, Large Magellanic Cloud Cepheid Standards Provide a 1\% Foundation for the Determination of the Hubble Constant and Stronger Evidence for Physics beyond $\Lambda$ CDM, ApJ 876 (May, 2019) 85, [arXiv: 1903.0760].

[25] P. S. Pasquini and O. L. G. Peres, Bounds on neutrino-scalar Yukawa coupling, Phys. Rev. D 93 (Mar, 2016) 053007, [arXiv: 1511.0181].

[26] J. M. Berryman, A. de Gouvêa, K. J. Kelly, and Y. Zhang, Lepton-number-charged scalars and neutrino beamstrahlung, Phys. Rev. D 97 (Apr, 2018) 075030, [arXiv:1802.0000].

[27] R. V. Wagoner, W. A. Fowler, and F. Hoyle, On the Synthesis of elements at very high temperatures, Astrophys.J. 148 (1967) 3-49.

[28] A. D. Dolgov, Neutrinos in cosmology, Phys. Rep. 370 (Nov., 2002) 333-535, [hep-ph/0202122].

[29] S. Weinberg, Cosmology. Oxford University Press, 2008.

[30] R. H. Cyburt, B. D. Fields, K. A. Olive, and T.-H. Yeh, Big bang nucleosynthesis: Present status, Reviews of Modern Physics 88 (Jan., 2016) 015004, [arXiv:1505.0107].

[31] E. Grohs, G. M. Fuller, C. T. Kishimoto, M. W. Paris, and A. Vlasenko, Neutrino energy transport in weak decoupling and big bang nucleosynthesis, Phys. Rev. D 93 (Apr., 2016) 083522, [arXiv: 1512.0220].

[32] L. Kawano, Let's go: Early universe 2. Primordial nucleosynthesis the computer way, NASA STI/Recon Technical Report 92 (Jan., 1992) 25163.

[33] A. R. Howe, E. Grohs, and F. C. Adams, Nuclear processes in other universes: Varying the strength of the weak force, Phys. Rev. D 98 (Sep, 2018) 063014, [arXiv:1809.0512].

[34] E. Grohs and G. M. Fuller, Insights into neutrino decoupling gleaned from considerations of the role of electron mass, Nuclear Physics B 923 (Oct., 2017) 222-244, [arXiv:1706.0339].

[35] A. F. Heckler, Astrophysical applications of quantum corrections to the equation of state of a plasma, Phys. Rev. D 49 (Jan., 1994) 611-617.

[36] N. Fornengo, C. W. Kim, and J. Song, Finite temperature effects on the neutrino decoupling in the early Universe, Phys. Rev. D 56 (Oct., 1997) 5123-5134, [hep-ph/9702324]. 
[37] J. J. Bennett, G. Buldgen, M. Drewes, and Y. Y. Y. Wong, Towards a precision calculation of the effective number of neutrinos $N_{\mathrm{eff}}$ in the Standard Model I: The QED equation of state, arXiv e-prints (Nov, 2019) arXiv:1911.04504, [arXiv:1911.0450].

[38] E. Grohs and G. M. Fuller, The surprising influence of late charged current weak interactions on Big Bang Nucleosynthesis, Nuclear Physics B 911 (Oct., 2016) 955-973, [arXiv:1607.0279].

[39] O. Pisanti, A. Cirillo, S. Esposito, F. Iocco, G. Mangano, G. Miele, and P. D. Serpico, PArthENoPE: Public algorithm evaluating the nucleosynthesis of primordial elements, Computer Physics Communications 178 (June, 2008) 956-971, [arXiv:0705.0290].

[40] A. Arbey, AlterBBN: A program for calculating the BBN abundances of the elements in alternative cosmologies, Computer Physics Communications 183 (Aug., 2012) 1822-1831, [arXiv:1106.1363].

[41] C. Pitrou, A. Coc, J.-P. Uzan, and E. Vangioni, Precision big bang nucleosynthesis with improved Helium-4 predictions, Phys. Rep. 754 (Sep, 2018) 1-66, [arXiv:1801.0802].

[42] E. Grohs, G. M. Fuller, C. T. Kishimoto, and M. W. Paris, Probing neutrino physics with a self-consistent treatment of the weak decoupling, nucleosynthesis, and photon decoupling epochs, J. Cosmology Astropart. Phys. 5 (May, 2015) 017, [arXiv:1502.0271].

[43] Planck Collaboration, N. Aghanim, Y. Akrami, M. Ashdown, J. Aumont, C. Baccigalupi, M. Ballardini, A. J. Banday, R. B. Barreiro, N. Bartolo, and et al., Planck 2018 results. VI. Cosmological parameters, ArXiv e-prints (July, 2018) [arXiv:1807.0620].

[44] M. Escudero, Precision Early Universe Thermodynamics made simple: $N_{\text {eff }}$ and Neutrino Decoupling in the Standard Model and beyond, arXiv e-prints (Jan, 2020) arXiv:2001.04466, [arXiv: 2001.0446].

[45] E. Aver, K. A. Olive, R. L. Porter, and E. D. Skillman, The primordial helium abundance from updated emissivities, J. Cosmology Astropart. Phys. 11 (Nov., 2013) 17.

[46] R. J. Cooke, M. Pettini, and C. C. Steidel, One Percent Determination of the Primordial Deuterium Abundance, ApJ 855 (Mar., 2018) 102, [arXiv:1710.1112].

[47] J. Froustey and C. Pitrou, Incomplete neutrino decoupling effect on big bang nucleosynthesis, Phys. Rev. D 101 (Feb., 2020) 043524, [arXiv:1912.0937].

[48] S. Mukohyama, Brane-world solutions, standard cosmology, and dark radiation, Physics Letters B 473 (Feb., 2000) 241-245, [hep-th/9911165].

[49] CMB-S4 Collaboration, J. E. Carlstrom et al., CMB-S4, in BAAS, vol. 51, p. 209, Sep, 2019. arXiv: 1908.0106.

[50] S. H. Hansen, G. Mangano, A. Melchiorri, G. Miele, and O. Pisanti, Constraining neutrino physics with big bang nucleosynthesis and cosmic microwave background radiation, Phys. Rev. D 65 (Dec., 2001) 023511, [astro-ph/0105385].

[51] J. Hamann, J. Lesgourgues, and G. Mangano, Using big bang nucleosynthesis in cosmological parameter extraction from the cosmic microwave background: a forecast for PLANCK, J. Cosmology Astropart. Phys. 3 (Mar., 2008) 4.

[52] J. P. Kneller and G. Steigman, BBN for pedestrians, New Journal of Physics 6 (Sept., 2004) 117, [astro-ph/0406320].

[53] E. Grohs, G. M. Fuller, C. T. Kishimoto, and M. W. Paris, Lepton asymmetry, neutrino spectral distortions, and big bang nucleosynthesis, Phys. Rev. D 95 (Mar., 2017) 063503, [arXiv: 1612.0198].

[54] A. D. Dolgov, S. H. Hansen, S. Pastor, S. T. Petcov, G. G. Raffelt, and D. V. Semikoz, Cosmological bounds on neutrino degeneracy improved by flavor oscillations, Nucl. Phys. B 632 (June, 2002) 363-382, [hep-ph/0201287]. 
[55] E. Castorina, U. França, M. Lattanzi, J. Lesgourgues, G. Mangano, A. Melchiorri, and S. Pastor, Cosmological lepton asymmetry with a nonzero mixing angle $\theta_{13}$, Phys. Rev. D 86 (July, 2012) 023517, [arXiv:1204.2510].

[56] L. Johns, M. Mina, V. Cirigliano, M. W. Paris, and G. M. Fuller, Neutrino flavor transformation in the lepton-asymmetric universe, Phys. Rev. D 94 (Oct, 2016) 083505, [arXiv: 1608.0133].

[57] S. Bashinsky and U. Seljak, Signatures of relativistic neutrinos in CMB anisotropy and matter clustering, Phys. Rev. D 69 (Apr, 2004) 083002, [astro-ph/0310198].

[58] B. Follin, L. Knox, M. Millea, and Z. Pan, First Detection of the Acoustic Oscillation Phase Shift Expected from the Cosmic Neutrino Background, Phys. Rev. Lett. 115 (Aug, 2015) 091301, [arXiv: 1503.0786].

[59] CMB-S4 Collaboration, J. E. Carlstrom et al., CMB-S4 Science Book, First Edition, ArXiv e-prints (Oct., 2016) [arXiv: 1610.0274].

[60] M. Archidiacono and S. Hannestad, Updated constraints on non-standard neutrino interactions from Planck, J. Cosmology Astropart. Phys. 2014 (July, 2014) 046, [arXiv:1311.3873].

[61] S. R. Elliott, A. A. Hahn, and M. K. Moe, Direct evidence for two-neutrino double-beta decay in ${ }^{82}$ Se, Phys. Rev. Lett. 59 (Nov, 1987) 2020-2023.

[62] T. Brune and H. Päs, Massive Majorons and constraints on the Majoron-neutrino coupling, Phys. Rev. D 99 (May, 2019) 096005, [arXiv:1808.0815].

[63] K. M. Belotsky, A. L. Sudarikov, and M. Yu. Khlopov, Constraint on anomalous $4 n u$ interaction, Phys. Atom. Nucl. 64 (2001) 1637-1642. [Yad. Fiz.64,1718(2001)].

[64] R. V. Konoplich and M. Yu. Khlopov, Constraints on triplet Majoron model due to observations of neutrinos from stellar collapse, Sov. J. Nucl. Phys. 47 (1988) 565-566. [Yad. Fiz.47,891(1988)].

[65] R. M. Bionta, G. Blewitt, C. B. Bratton, D. Casper, A. Ciocio, R. Claus, B. Cortez, M. Crouch, S. T. Dye, S. Errede, G. W. Foster, W. Gajewski, K. S. Ganezer, M. Goldhaber, T. J. Haines, T. W. Jones, D. Kielczewska, W. R. Kropp, J. G. Learned, J. M. Losecco, J. Matthews, R. Miller, M. S. Mudan, H. S. Park, L. R. Price, F. Reines, J. Schultz, S. Seidel, E. Shumard, D. Sinclair, H. W. Sobel, J. L. Stone, L. R. Sulak, R. Svoboda, G. Thornton, J. C. van der Velde, and C. Wuest, Observation of a neutrino burst in coincidence with supernova 1987A in the Large Magellanic Cloud, Phys. Rev. Lett. 58 (Apr, 1987) 1494-1496.

[66] K. Hirata, T. Kajita, M. Koshiba, M. Nakahata, Y. Oyama, N. Sato, A. Suzuki, M. Takita, Y. Totsuka, T. Kifune, T. Suda, K. Takahashi, T. Tanimori, K. Miyano, M. Yamada, E. W. Beier, L. R. Feldscher, S. B. Kim, A. K. Mann, F. M. Newcomer, R. van, W. Zhang, and B. G. Cortez, Observation of a neutrino burst from the supernova SN1987A, Phys. Rev. Lett. 58 (Apr, 1987) 1490-1493.

[67] S. Shalgar, I. Tamborra, and M. Bustamante, Core-collapse supernovae stymie secret neutrino interactions, arXiv e-prints (Dec., 2019) arXiv:1912.09115, [arXiv:1912.0911].

[68] M. Wyman, D. H. Rudd, R. A. Vanderveld, and W. Hu, Neutrinos Help Reconcile Planck Measurements with the Local Universe, Phys. Rev. Lett. 112 (Feb, 2014) 051302, [arXiv: 1307.7715].

[69] A. V. Patwardhan and G. M. Fuller, Late-time vacuum phase transitions: Connecting sub-eV scale physics with cosmological structure formation, Phys. Rev. D 90 (Sep, 2014) 063009, [arXiv: 1401.1923].

[70] G. B. Gelmini, A. Kusenko, and V. Takhistov, Hints of Sterile Neutrinos in Recent Measurements of the Hubble Parameter, arXiv e-prints (Jun, 2019) arXiv:1906.10136, [arXiv: 1906.1013]. 
[71] V. E. Kuzmichev and V. V. Kuzmichev, Uncertainty principle in quantum mechanics with Newton's gravity, arXiv e-prints (Nov, 2019) arXiv:1911.01176, [arXiv:1911.0117].

[72] G. Mangano, G. Miele, S. Pastor, O. Pisanti, and S. Sarikas, Constraining the cosmic radiation density due to lepton number with Big Bang Nucleosynthesis, J. Cosmology Astropart. Phys. 3 (Mar., 2011) 035, [arXiv:1011.0916]. 